\title{
锂离子电池高电压和耐燃电解液研究进展
}

\author{
夏兰余林颇胡笛陈政* \\ (宁波诺丁汉大学 理工学院 宁波 315100)
}

\begin{abstract}
摘要 电解液作为锂离子电池的重要组成部分, 起着传输离子的作用, 电解液的性质对电池的容量、循环性能及安全 性能等影响巨大. 近年来, 随着高电压、高能量密度锂离子电池的开发应用, 现有常规碳酸酯电解液存在正极稳定性 差、闪点低、易燃烧等问题. 因此, 发展高电压耐燃电解液是应用高电压高容量正极材料、发展高电压高容量高安全 性锂离子电池的迫切需要. 主要综述了高电压电解液、耐燃性电解液及兼具抗氧化性和耐燃性的高浓度电解液的研究 进展和现状. 在此基础上, 对锂离子电池新型电解液的发展方向进行了展望.
\end{abstract}

关键词 锂离子电池; 电解液; 高电压; 耐燃; 高浓度

\section{Research Progress and Perspectives on High Voltage, Flame Retardant Electrolytes for Lithium-Ion Batteries}

\author{
Xia, Lan Yu, Linpo Hu, Di Chen, Z. George* \\ (Faculty of Science and Engineering, University of Nottingham Ningbo China, Ningbo 315100)
}

\begin{abstract}
The electrolyte is an indispensable constituent in lithium ion batteries, and its role conducts electricity by means of the transportation of charge carries between the pair of electrodes. Its properties directly affect the energy density, cycle life and safety of the battery. However, there are two major challenges to using carbonate-based electrolytes in recent lithium ion batteries (LIBs) to further increase the energy density of the devices without compromising the safety. One is that carbonate-based electrolytes are not sufficiently stable at the positive electrode, and the other is their relatively high flammability. Therefore, developing high voltage and flame retardant electrolytes for LIBs is highly desired. Herein, we review the recent progress and challenges in new electrolytes, focusing on high-voltage electrolytes, flame retardant electrolytes and highly concentrated electrolytes. Among the reported electrolytes, highly concentrated electrolytes are worth a special attention, showing various unusual functionalities, for example, high oxidative stability, low volatility, high reductive stability, and non-corrosive to Al. These special properties are totally different from that of the conventional $1 \mathrm{~mol} \cdot \mathrm{L}^{-1} \mathrm{LiPF}_{6} / \mathrm{EC}$-based electrolytes, which are result from solution structures. A discussion is also provided in this review on the prospects of further development of new electrolytes for LIBs.
\end{abstract}

Keywords lithium ion battery; electrolyte; high voltage; flame retardant; highly concentrated

\section{1 引言}

目前, 商品化锂离子电池所采用的电解液由有机溶 剂以及溶于其中的 $\mathrm{LiPF}_{6}{ }^{[1,2]}$ 所组成. 其中, 有机溶剂通 常是以介电常数大的碳酸乙烯酯(Ethylene carbonates, $\mathrm{EC})^{[3]}$ 为基础的二元或三元混合溶剂, 其中, 链状碳酸 酯(碳酸二甲酯 DMC、碳酸二乙酯 DEC 或碳酸甲乙酯 $\mathrm{EMC}) 、$ 羧酸酯或梄类为共溶剂 ${ }^{[4]}$. 这些常用溶剂具有与 锂盐的溶解性好、所组成的电解液电导率高、且能够在 正负极形成稳定的 SEI 膜等特点, 因而被认为是电解液 体系溶剂的最佳选择. 然而, 在锂离子电池的开发及应 用的过程中, 常用碳酸酯电解液遭遇到以下两个方面的
问题(如图 1).

其一, 常规碳酸酯电解液正极稳定性差、抗氧化能 力不强 ${ }^{[5,6]}$, 严重阻碍了高电压正极材料的开发. 为了进 一步提升锂离子电池的能量密度, 人们开发了具有较高 工作电压的正极材料, 主要包括高电压 $\mathrm{LiNi}_{0.5} \mathrm{Mn}_{1.5} \mathrm{O}_{4}$ 、 $\mathrm{LiMPO}_{4}(\mathrm{M}=\mathrm{Mn} 、 \mathrm{Co} 、 \mathrm{~V}) 、 \mathrm{Li}_{2} \mathrm{MPO}_{4} \mathrm{~F}(\mathrm{M}=\mathrm{Ni} 、 \mathrm{Co})$ 和 富锂锰基固溶体 $x \mathrm{Li}_{2} \mathrm{MnO}_{3} \cdot(1-x) \mathrm{LiMeO}_{2}(\mathrm{Me}=\mathrm{Ni} 、 \mathrm{Co}$ 、 $\mathrm{Mn})$ 等 ${ }^{[7]}$, 它们的充电截止电压都在 $4.7 \mathrm{~V}$ 以上, 而常规 的碳酸酯电解液在电位达到 $4.5 \mathrm{~V}$ 时在高氧化活性的正 极材料表面发生不可逆的氧化分解, 生成 $\mathrm{CO}_{2} 、 \mathrm{H}_{2} \mathrm{O}$ 和 聚碳酸乙烯基(PEC) 等产物, 引起电池胀气、电极界面组 成变化、极化增大等, 从而导致高电压正极材料循环性

*E-mail: george.chen@nottingham.edu.cn

Received June 28, 2017; published September 4, 2017.

Project supported by the National Natural Science Foundation of China (No. 21503246), the Ningbo Municipal Government (3315 Plan and the IAMET Special Fund, No. 2014A35001-1), Ningbo Natural Science Foundation Programme (Nos. 2017A610022, 2016A610114, 2016A610115), and the Project of Science and Technology Department of Zhejiang Province (Nos. 2016C31023、No. 2017C31104).

项目受国家自然科学基金(No. 21503246)、宁波市科技项目(“3315 计划”、No. 2014A35001-1), 宁波市自然科学基金(Nos. 2017A610022, 2016A610114, 2016A610115)和浙江省科技厅项目(Nos. 2016C31023, 2017C31104)资助. 


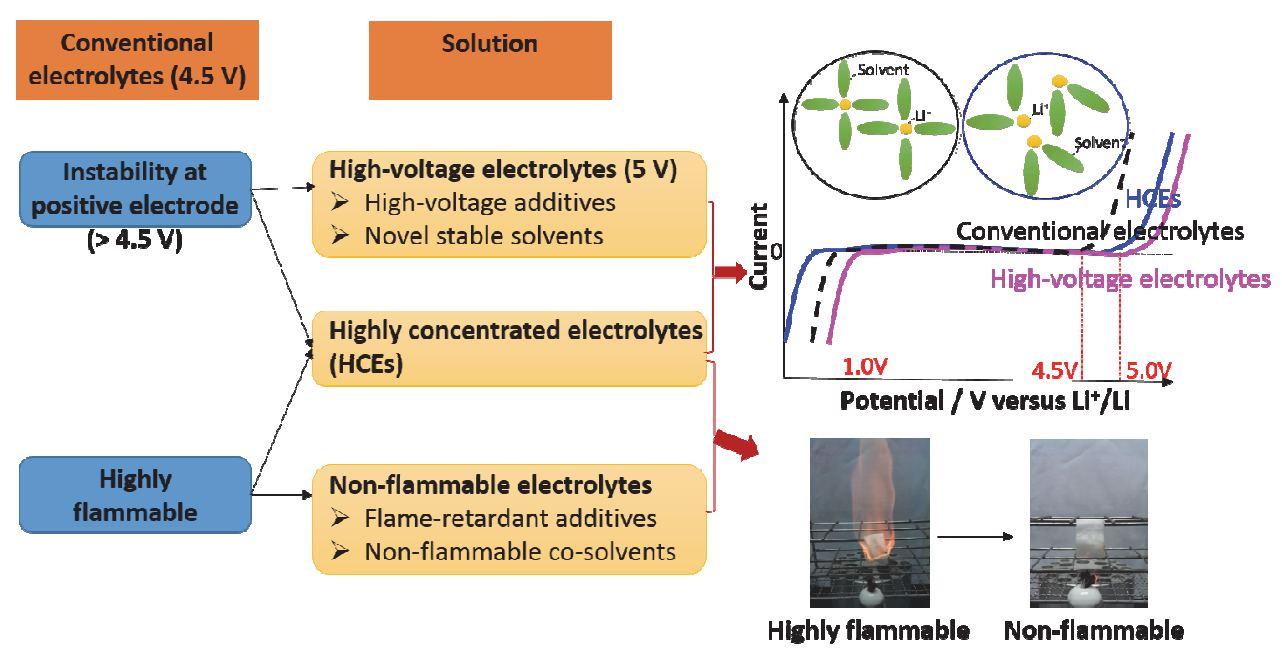

图 1 锂离子电池常规电解液的两个问题及解决措施

Figure 1 Problems and their solutions for the conventional electrolytes in lithium-ion batteries

能明显恶化等问题 ${ }^{[8 \sim 11]}$. 因此, 开发具有正极稳定性 高、抗氧化性好的高电压电解液是发展高电压正极材料 的迫切需要.

其二, 常用的有机碳酸酯电解液的低闪点和易燃性 是锂离子电池发生燃烧、爆炸事故的主要原因, 因此, 降低电解液可燃性是提高锂离子电池安全性的有效途 径. 传统的电解液体系除了含有锂盐 $\mathrm{LiPF}_{6}$ 、高介电常 数的环状碳酸乙烯酯 EC 外, 还包括低沸点、低粘度的 线性碳酸酯溶剂如 DMC、DEC 或 EMC 等, 它们的闪点 通常较低, 分别为 $17 、 33$ 和 $23{ }^{\circ} \mathrm{C}$ (表 1 为常用的碳酸 酯溶剂的熔沸点、闪点的比较). 当电池处于过充电、短 路、高热冲击等滥用的情况下, 电池内部的温度和压力 均很高, 碳酸酯电解液容易被点燃, 很可能引起电池发 生燃烧、甚至爆炸等危险 ${ }^{[4,12 \sim 16]}$. 因此, 寻找具有高闪点 的共溶剂、发展耐燃电解液是提高锂离子电池电压和能 量密度的同时，保证安全性的有效途径.

表 1 常用的碳酸酯溶剂的熔沸点、闪点比较

Table 1 Comparisons of physical properties of conventional carbonates

\begin{tabular}{lccccc}
\hline \multicolumn{1}{c}{ Solvent } & DMC & DEC & EMC & EC & PC \\
\hline Melting point $/{ }^{\circ} \mathrm{C}$ & 4.6 & -74.3 & -53 & 36.4 & -48.8 \\
Boiling point $/{ }^{\circ} \mathrm{C}$ & 91 & 126 & 110 & 248 & 242 \\
Flash point $/{ }^{\circ} \mathrm{C}$ & $\mathbf{1 7}$ & $\mathbf{3 3}$ & $\mathbf{2 3}$ & 160 & 132 \\
\hline
\end{tabular}

综上所述, 研发高电压耐燃电解液作为大容量锂离 子电池的关键材料是推动电动汽车、储能电站等新能源 技术领域商业化应用的迫切需求.

\section{2 高电压电解液}

开发高电压电解液的技术途径主要有两种, 一是在 电解液中加入少量具有针化正极界面膜作用的高电压 添加剂; 二是用具有抗氧化能力强的新型溶剂来全部或 部分替代现有的抗氧化性不高的碳酸酯溶剂, 如二腈 类、砜类、离子液体和氟代溶剂等 ${ }^{[17]}$.

\section{1 高电压添加剂}

在电池的首周充电过程中, 添加剂分子参与并改善 正极材料表面界面膜性质, 形成钝化的正极界面膜, 阻 断电解液与正极表面的直接接触, 抑制电解液组分在充 电状态下正极表面的氧化分解. 目前研究的此类添加剂 (各物质的结构式如图 2 所示)包括: 含草酸根离子的锂 盐, 如二草酸硼酸锂 $(\mathrm{LiBOB})^{[18,19]}$ 和二氟草酸硼酸锂 $(\mathrm{LiDFOB})^{[20]}$; 含磷化合物, 三甲基磷酸酯 $(\mathrm{TMP})^{[21]}$, 三 $(1,1,1,3,3,3 \text {-六氟异丙基)磷酸酯(HFiP) })^{[22,23]}$,三(三甲基 硅)磷酸酯(TMSP) ${ }^{[24 ~ 27]}$ 和 1-丙基磷酸环䣶(PACA $)^{[28]}$ 等; 一些磺酸酯, 如 1,3-丙烷磺酸内酯(PS)和硫酸亚丙酯 $(\mathrm{PCS})^{[29]}$, 以及一些酸酐类化合物 ${ }^{[30 ~ 32]}$ 等.

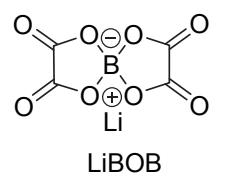<smiles>O=C1O[Se](F)(F)C(=O)C1=O</smiles>
LiDFOB<smiles>COP(=O)(OC)O[Na]</smiles><smiles>O=P(OC(C(F)(F)F)C(F)(F)F)(OC(F)(F)C(F)(F)F)OC(F)(F)C(F)(F)F</smiles><smiles>C[Si](C)(C)OP(=O)(O[Si](C)(C)C)O[Si](C)(C)C</smiles><smiles>CCCP1(=O)OP(=O)(CCC)OP(=O)(CCC)O1</smiles>
PACA

图 2 一些高电压添加剂的结构式

Figure 2 Chemical structures of some high voltage additives

LiBOB 作为高电压添加剂, 其作用机理是: 首周充 电时, LiBOB 会在正极材料表面发生氧化分解、聚合反 应, 形成阻抗小、厚度薄的正极界面膜, 阻止电解液中 溶剂的进一步氧化，从而明显地提高高电压正极材料的 循环稳定性 ${ }^{[18]}$. 同时, $\mathrm{LiBOB}$ 因其阴离子 $\mathrm{BOB}^{-}$在 $1.7 \mathrm{~V}$ vs. $\mathrm{Li}^{+} / \mathrm{Li}$ 时发生还原分解, 容易在负极表面形成稳定 的 SEI 膜, 故其也可以作为负极添加剂用 ${ }^{[33,34]}$. 与 LiBOB 相比, LiDFOB 形成的界面膜更稳定且阻抗更低, 
其原因在于 $\mathrm{LiBOB}$ 中的一个草酸根被两个 $\mathrm{F}$ 取代后, 分

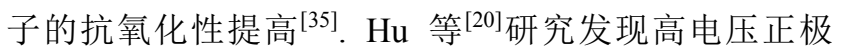
$\mathrm{LiCoPO}_{4}$ 在含 LiDFOB 添加剂电解液中显示出更好的循 环性能. XPS 和 FTIR-ATR 分析结果证实, 正极表面存 在 $\mathrm{B}$ 元素、且几乎没有发现聚碳酸乙烯衍生物(PEC)的 存在, 表明 LiDFOB 参与形成了稳定的界面膜, 大大降 低了电解液中 EC 的氧化聚合 ${ }^{[19]}$.

最近, Lucht 等 ${ }^{[36]}$ 设计并合成了四个新型的烷基/芳 基三甲基嗍酸锂, 并考察了它们作为添加剂用于高电压 石墨 $/ \mathrm{LiNi}_{0.5} \mathrm{Mn}_{1.5} \mathrm{O}_{4}$ 电池. 图 3 为石墨 $/ \mathrm{LiNi}_{0.5} \mathrm{Mn}_{1.5} \mathrm{O}_{4}$ 电 池在不同添加剂电解液中的室温和高温 $55{ }^{\circ} \mathrm{C}$ 循环性能 比较. 从图中可以看出, 在 $1 \mathrm{~mol} \cdot \mathrm{L}^{-1} \mathrm{LiPF}_{6} / \mathrm{EC}+\mathrm{EMC}$ 电解液中添加 $0.5 \sim 2.0 \mathrm{wt} \%$ 的烷基/芳基三甲基硼酸锂 可以明显改善高电压电池的循环稳定性和库伦效率. 通 过 TEM、SEM、XPS 和 IR-ATR 等非现场表征技术研究 发现，4-吡啶三甲基嗍酸锂(LPTB)同时在正极和负极表 面反应, 形成稳定的界面膜. 具体的作用机理为: 四烷 基嗍酸阴离子 $\mathrm{B}(\mathrm{OR})_{4}^{-}$被正极表面的氧化物 $\mathrm{M}_{x} \mathrm{O}_{y}$ 氧化 生成复合物 $\mathrm{M}_{x} \mathrm{O}_{y}-\mathrm{B}(\mathrm{OR})_{4}$, 该复合物的形成有效阻止了 溶剂分子的氧化分解和金属 $\mathrm{Mn} / \mathrm{Ni}$ 的溶解流失, 从而明 显地改善了电池的电化学性能.

从目前研究进展来看, 高电压添加剂是从动力学角 度出发, 通过在正极表面形成稳定的界面膜, 起到钝化 作用, 从而抑制电解液中溶剂的不可逆氧化分解, 提高 电池的高电压循环稳定性. 然而, 研究者们甚少考察实 际电池的自放电行为, 而电池的自放电与电解液及其正 极界面膜在高电位下氧化分解息息相关. Passerini 等 ${ }^{[31]}$ 系统考察了 FEC、 LiBOB 等已报道的 40 种高电压添加 剂在 $\mathrm{Li} / \mathrm{LiNi}_{0.4} \mathrm{Mn}_{1.6} \mathrm{O}_{4}$ 电池中的自放电行为. 研究发现, 在这 40 种添加剂中只有丁二酸酐 $(\mathrm{SA})$ 能够减小自放电、 电压降和容量损失, 同时提高电池的库伦效率. 而其它 常用高电压添加剂如 LiBOB、LiDFOB 以及丁二酸酐类 衍生物却恶化了电池的自放电行为. 由此可见, 高电压 添加剂的实际应用需要综合考察的各方面因素很多, 包 括高低温循环性能、库伦效率以及自放电情况等.

\section{2 抗氧化性的新型溶剂}

新型的抗氧化溶剂也是开发高电压电解液的重要 方向之一. 目前研究的抗氧化性溶剂主要包括二腈类、 砜类、离子液体和氟代溶剂, 人们对这四类抗氧化性溶 剂用于高电压电解液的可行性进行了大量深入的探索.

早在 1994 年日本三菱公司 Ue 等 ${ }^{[37,38]}$ 报道了各种有 机溶剂应用于双电层电容器体系的可能性. 结果发现, 二腈类溶剂如戊二腈(GLN)和已二腈(AND)的电化学窗 口宽达 $8 \mathrm{~V}$ 以上, 氧化分解电位为约 $8.3 \mathrm{~V} \mathrm{vs.} \mathrm{Li}^{+} / \mathrm{Li}$, 显 示出超高的正极稳定性. 虽然纯二腈类溶剂具有抗氧化 能力强、介电常数大且沸点闪点高等优点, 但其存在熔 点高、粘度大、对隔膜的浸润能力差等缺点, 使其难以 作为纯溶剂使用, 需要配合碳酸酯类溶剂混合使用. 二
腈类溶剂作为共溶剂时，随着其体积百分比含量的增 加, 电解液的氧化分解电位越高 ${ }^{[39]}$.
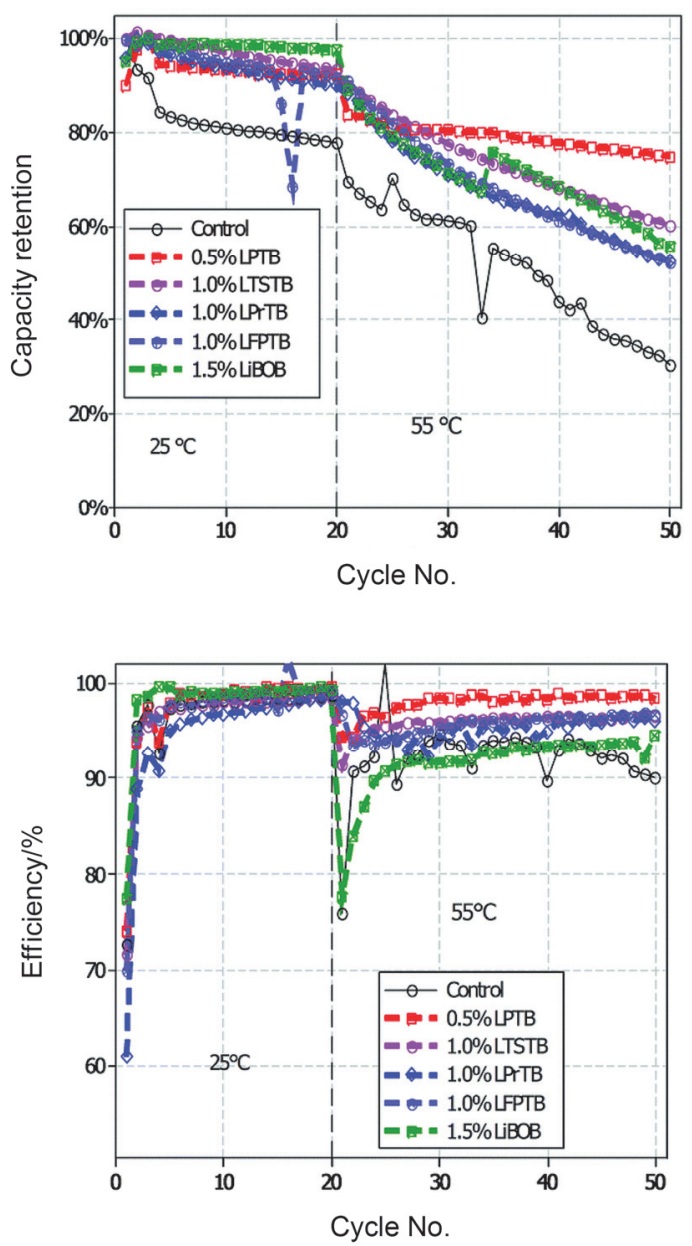

图 3 石墨 $/ \mathrm{LiNi}_{0.5} \mathrm{Mn}_{1.5} \mathrm{O}_{4}$ 电池在含不同添加剂电解液中的室温和高 温循环性能比较 ${ }^{[36]}$

Figure 3 Cycling performance of graphite/ $\mathrm{LiNi}_{0.5} \mathrm{Mn}_{1.5} \mathrm{O}_{4}$ cells at $25{ }^{\circ} \mathrm{C}$ and $55{ }^{\circ} \mathrm{C}$ with the baseline electrolyte with and without added lithium 4-pyridyl trimethyl borate $(\mathrm{LPTB})^{[36]}$

Nagahama 等 ${ }^{[40]}$ 考察了高电压正极材料 $\mathrm{Li}_{2} \mathrm{Ni}_{0.98} \mathrm{Co}_{0.02} \mathrm{PO}_{4} \mathrm{~F}$ 在 $1 \mathrm{~mol} \cdot \mathrm{L}^{-1} \mathrm{LiBF}_{4} / \mathrm{EC}+\mathrm{DMC}+$ 癸二 腈电解液 $(25: 25: 50$, by vol.)中的充放电曲线, 首次观 察到 $5.3 \mathrm{~V}$ 的充电电压平台, 但未给出其循环性能. 因 此, 二腈类溶剂可以作为高电压电解液的溶剂, 但是因 其含 $\mathrm{C}=\mathrm{N}$ 双键而极易在石墨或金属锂等低电位负极表 面发生聚合反应，生成的聚合产物会阻止 $\mathrm{Li}^{+}$的脱嵌， 进而引起电池容量的衰减 ${ }^{[41]}$. 为了提高二腈类电解液 在石墨负极的稳定性, 研究者们通过在电解液中加入 $\mathrm{LiBOB}^{[42,43]}$ 、碳酸亚乙烯酯(VC)或 $\mathrm{FEC}^{[44]}$ 等成膜添加剂, 形成稳定的 SEI 膜, 以期改善负极稳定性, 但实际的应 用效果不太明显.

砜类是另一类具有抗氧化性的新型溶剂. 1998 年, Angell 等 ${ }^{[45]}$ 考察了非环状脂肪族砜类化合物甲乙砜 (EMSF)作为新型溶剂的性能, 与醚类、碳酸酯类相比, EMSF 具有宽的电化学窗口, 氧化分解电位达 $5.89 \mathrm{~V}$ vs. 
$\mathrm{Li}^{+} / \mathrm{Li}$, 可以作为高电压电解液的溶剂. 但 EMSF 作溶 剂或共溶剂的高电压电解液均会导致石墨的剥离. 为了 阻止砜在石墨中的共嵌入而破坏石墨结构, Angell 等 ${ }^{[46,47]}$ 在电解液中引入了几种含环戊基(ESCP)及环己 基取代的砜类溶剂, 这些电解液的电化学窗口超过 5.5 $\mathrm{V}$ vs. $\mathrm{Li}^{+} / \mathrm{Li}$, 但电导率比 EMSF 作溶剂的电解液低一个 数量级. $\mathrm{Li} / \mathrm{MCMB}$ 及 $\mathrm{Li} / \mathrm{LiCr}_{0.015} \mathrm{Mn}_{1.985} \mathrm{O}_{4}$ 半电池测试显 示, MCMB 在 $1 \mathrm{~mol} \cdot \mathrm{L}^{-1} \mathrm{LiTFSI} / \mathrm{ESCP}$ 的电解液中容量 衰减很快, 而正极循环 120 周后库仑效率保持在 $86 \%$. 由此可知, 砜类溶剂对石墨类碳材料兼容性差, 在正极 上的库仑效率也不高, 因此, 寻找合适的成膜剂是提高 砜类溶剂的应用的关键. 在砜类电解液中加入成膜添加 剂如 $\mathrm{VC}^{[48 \sim 50]}$ 、 $\mathrm{LiBOB}^{[51,52]}$ 、对甲苯磺酰异氭酸酯 $(\mathrm{PTSI})^{[53]}$ 和六亚甲基二异氰酸酯(HDI) ${ }^{[54]}$ 促进稳定 SEI 膜的形成, 获得与常规碳酸酯电解液相当的电化学性 能. 随后, Amine 等 ${ }^{[55]}$ 研究了无需形成 SEI 膜的负极材 料 $\mathrm{Li}_{4} \mathrm{Ti}_{5} \mathrm{O}_{12}$ 在砜类溶剂中的性能, 他们在环丁砜(TMS) 中加入等体积的 $\mathrm{EMC}$ 为共溶剂, 在 $2 \mathrm{C}$ 下循环了 1000 周后没有明显的容量损失. Xing 等 ${ }^{[56]}$ 利用分子动力学模 拟研究了电解液中各溶剂组分在正负极表面的存在状 态. 结果显示, $1 \mathrm{~mol} \cdot \mathrm{L}^{-1} \mathrm{LiPF}_{6} / \mathrm{TMS}+\mathrm{DMC}$ 电解液中的 溶剂组分 $\mathrm{DMC}$ 离正极界面的距离比 $1 \mathrm{~mol} \cdot \mathrm{L}^{-1}$ $\mathrm{LiPF}_{6} / \mathrm{EC}+\mathrm{DMC}$ 电解液中远, 这可能是其电解液氧化 稳定性提高的原因所在.

室温离子液体完全由阴、阳离子两部分组成, 阴离 子通常有 $\mathrm{PF}_{6}^{-}$、 $\mathrm{TFSI}^{-}$等, 阳离子包括季铵型、咪唑型 和吡啶型等. 它们具有挥发性极小、不燃、电化学窗口 宽、溶解能力强等特点, 常被用来作为锂离子电池高性 能电解液溶剂 ${ }^{[57 ~ 63]}$. Borgel 等 ${ }^{[64]}$ 考察了不同类型的离子 液体应用于高电压正极 $\mathrm{LiNi}_{0.5} \mathrm{Mn}_{1.5} \mathrm{O}_{4}$ 的可行性. 研究 发现, 咪唑型及季铵型离子的氧化电位都在 $5 \mathrm{~V}$ 以上, 但只有在环状季铵型离子液体中可以可逆的沉积锂; $\mathrm{LiNi}_{0.5} \mathrm{Mn}_{1.5} \mathrm{O}_{4}$ 正极在环状季铵型离子液体 $\mathrm{PP}_{13}$-TFSI 与 $\mathrm{PP}_{14}$-TFSI 中可以正常充放电, 且库仑效率均比在碳酸 酯类电解液中的高. 而 $\mathrm{LiNi}_{0.5} \mathrm{Mn}_{1.5} \mathrm{O}_{4}$ 电极在咪唑型离 子液体及四烷基季铵型离子液体中循环 5 周后, 放电容 量急剧衰减. 并且, 离子液体因自身粘度较大, 导致电 导率低, 需要配合使用其它溶剂. Xiang 等 ${ }^{[65]}$ 报道了 $\mathrm{PP}_{14}$-TFSI 和环丁砜 TMS 混合使用的电解液应用于高电 压电池. 结果显示, $0.5 \mathrm{~mol} \cdot \mathrm{L}^{-1} \mathrm{LiDFOB} /(60 \%) \mathrm{PP}_{14^{-}}$ TFSI/(40\%)TMS 电解液的室温电导率较高 $\left(10^{-3} \mathrm{~S} \cdot \mathrm{cm}^{-1}\right)$ 且完全不燃, $\mathrm{Li}_{1.2} \mathrm{Ni}_{0.2} \mathrm{Mn}_{0.6} \mathrm{O}_{2}$ 电极在该电解液中电化学 性能明显提高. Mun 等 ${ }^{[66]}$ 分别研究了高电压正极 $\mathrm{LiNi}_{0.5} \mathrm{Mn}_{1.5} \mathrm{O}_{4}$ 在碳酸酯基和离子液体基电解液中形成 的界面膜的组成. 研究发现, 在碳酸酯基电解液中, 界 面膜组分中的无机物较多; 而在离子液体基电解液中, 界面膜组分以有机物为主.

由于 $\mathrm{F}$ 原子具有强吸电子性, 氟代溶剂具有比常规
溶剂更好的氧化稳定性, 目前已成为高电压电解液研究 的热点之一. 表 2 为氟代溶剂与其未氟代的常规溶剂的 HOMO 和 LUMO 能级比较. 从表 2 可以看出, 氟代溶剂 不仅 HOMO 能级较小、氧化分解电位高, 可以作为高电 压电解液的溶剂, 而且 LUMO 能级也随之降低、还原电 位正移，容易被还原。早在 2010 年，日本 Kitagawa 等 ${ }^{[67]}$ 首次将氟代碳酸乙烯酯(FEC)和氟代醚—1, 1,2,2-四氟 乙基-2,2,3,3-四氟丙基醚(TFTFEP)用于高电压 $\mathrm{LiCoO}_{2}$ 正极. 研究表明, $1 \mathrm{~mol} \cdot \mathrm{L}^{-1} \mathrm{LiPF}_{6} / \mathrm{FEC}+\mathrm{TFTFEP}(1: 1$, by vol.) 氟代电解液(其中 FEC 介电常数大, 故电解液的 离子电导率高) 与常规电解液相比, $\mathrm{LiCoO}_{2}$ 正极在 $4.5 \sim$ $3.0 \mathrm{~V}$ 的充放电电压区间显示出更优异的循环稳定性. 近几年, Amine 等 ${ }^{[68]}$ 和 Markevich 等 ${ }^{[69]}$ 均报道了 FEC 基 氟代电解液明显提高了高电压锂离子电池的室温循环 性能. 随后, Amine 等 ${ }^{[70]}$ 在 $\mathrm{EC}$ 及 $\mathrm{EMC}$ 分子上引入含氟 烷基，配合使用氟代醚 TFTFEP 为共溶剂制备出全氟代 电解液. 高电压电池 $\mathrm{Li} / \mathrm{LiNi}_{0.5} \mathrm{Mn}_{1.5} \mathrm{O}_{4}$ 和 $\mathrm{Li}_{4} \mathrm{Ti}_{5} \mathrm{O}_{12} /$ $\mathrm{LiNi}_{0.5} \mathrm{Mn}_{1.5} \mathrm{O}_{4}$ 采用该氟代电解液后, 它们的常温及高温 循环性能得到明显提高.

表 2 氟代溶剂与其未氟代溶剂的 HOMO 和 LUMO 能级比较 ${ }^{[70,73]}$

Table 2 Comparison of some fluorinated solvents with its nonfluorinated counterpart solvents ${ }^{[70,73]}$

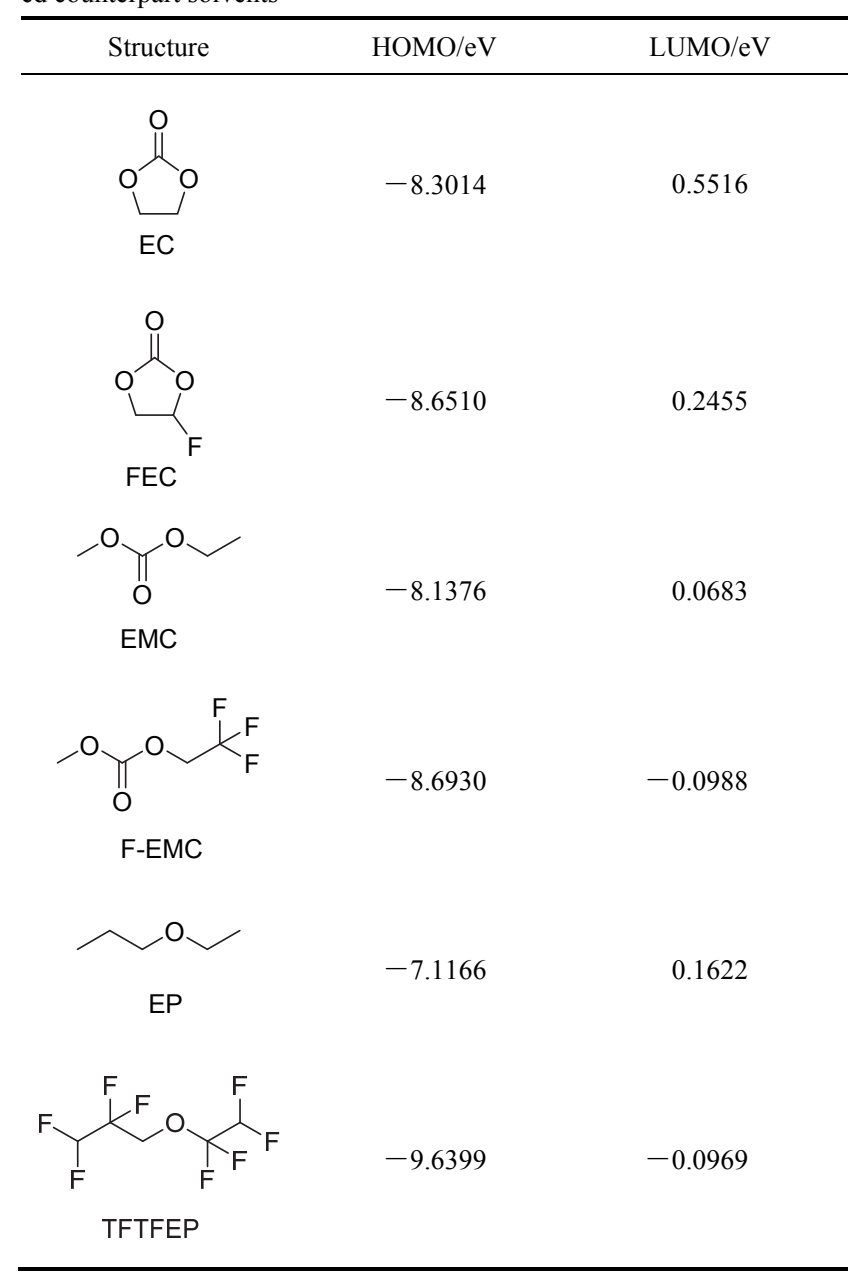


以金属锂片, $\mathrm{Li}_{4} \mathrm{Ti}_{5} \mathrm{O}_{12}$ 或 $\mathrm{Si}$ 为负极的高电压锂离子 电池在氟代电解液中具有较好的循环稳定性. 然而, 由 于氟化之后, 含氟溶剂的还原电位升高、易被还原, 导 致报道的氟代电解液与石墨类碳负极的兼容性较差, 导 致电池的循环性能不佳 ${ }^{[71]}$. 为了改善氟代电解液与石 墨负极的稳定性, 同时兼顾氟代溶剂的高电压性能, Xia 等 ${ }^{[72]}$ 引了具有还原电位较高, 但可稳定石墨负极 SEI 膜, 并具有成膜作用的氟代醚一 $1,1,1,3,3,3$-六氟异丙 基甲基醚 (HFPM) 作为共溶剂, 制备了 $1 \mathrm{~mol} \cdot \mathrm{L}^{-1}$ $\mathrm{LiPF}_{6} / \mathrm{FEC}+\mathrm{DMC}+\mathrm{EMC}+\operatorname{HFPM}(2: 3: 1: 4$, by vol. $)$ 氟代电解液. 研究发现(如图 4), 该电解液不仅浸润性 好、完全不燃, 而且装配该电解液的 $\mathrm{MCMB} /$
$\mathrm{LiNi}_{0.5} \mathrm{Mn}_{1.5} \mathrm{O}_{4}$ 实际高电压电池(型号 18650 , 容量 1500 $\mathrm{mAh}$ )显示了优异的循环性能(循环 200 周后，容量保持 率达 $82 \%$, 明显高于常规电解液的 $61 \%$ ), 并通过了针刺 实验, 展现出了较好的安全性能.

\section{3 耐燃性电解液}

众所周知, 有机碳酸酯电解液的低闪点和易燃性是 锂离子电池发生燃烧、爆炸等不安全事故的根本原

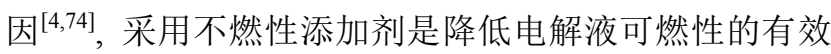
途径 ${ }^{[12,13]}$. 目前研究的不燃性添加剂或共溶剂主要包括 含氟溶剂 ${ }^{[75 ~ 79]}$ 、含磷化合物 ${ }^{[80]}$ 和离子液体 ${ }^{[81 \sim 83]}$ 等. a

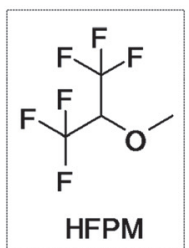

\begin{tabular}{ccc}
\hline Electrolyte & \multicolumn{1}{c}{ Composition (VIV) } & $\begin{array}{c}\text { Conductivity/ } \\
\left(\mathrm{mS} \cdot \mathrm{cm}^{-1}\right), \text { at } 30^{\circ} \mathrm{C}\end{array}$ \\
\hline $\begin{array}{l}\text { The base } \\
\text { electrolyte }\end{array}$ & $1 \mathrm{~mol} \cdot \mathrm{L}^{-1} \mathrm{LiPF}_{6} / \mathrm{EC}+\mathrm{DMC}(3: 7)$ & 11.56 \\
$\begin{array}{c}\text { F-based } \\
\text { electrolyte }\end{array}$ & $\begin{array}{l}1 \mathrm{~mol} \cdot \mathrm{L}^{-1} \mathrm{LiPF}_{6} / \mathrm{FEC}+\mathrm{DMC}+\mathrm{EMC}+\mathrm{HFPM} \\
(2: 3: 1: 4)\end{array}$ & 8.57 \\
\hline
\end{tabular}
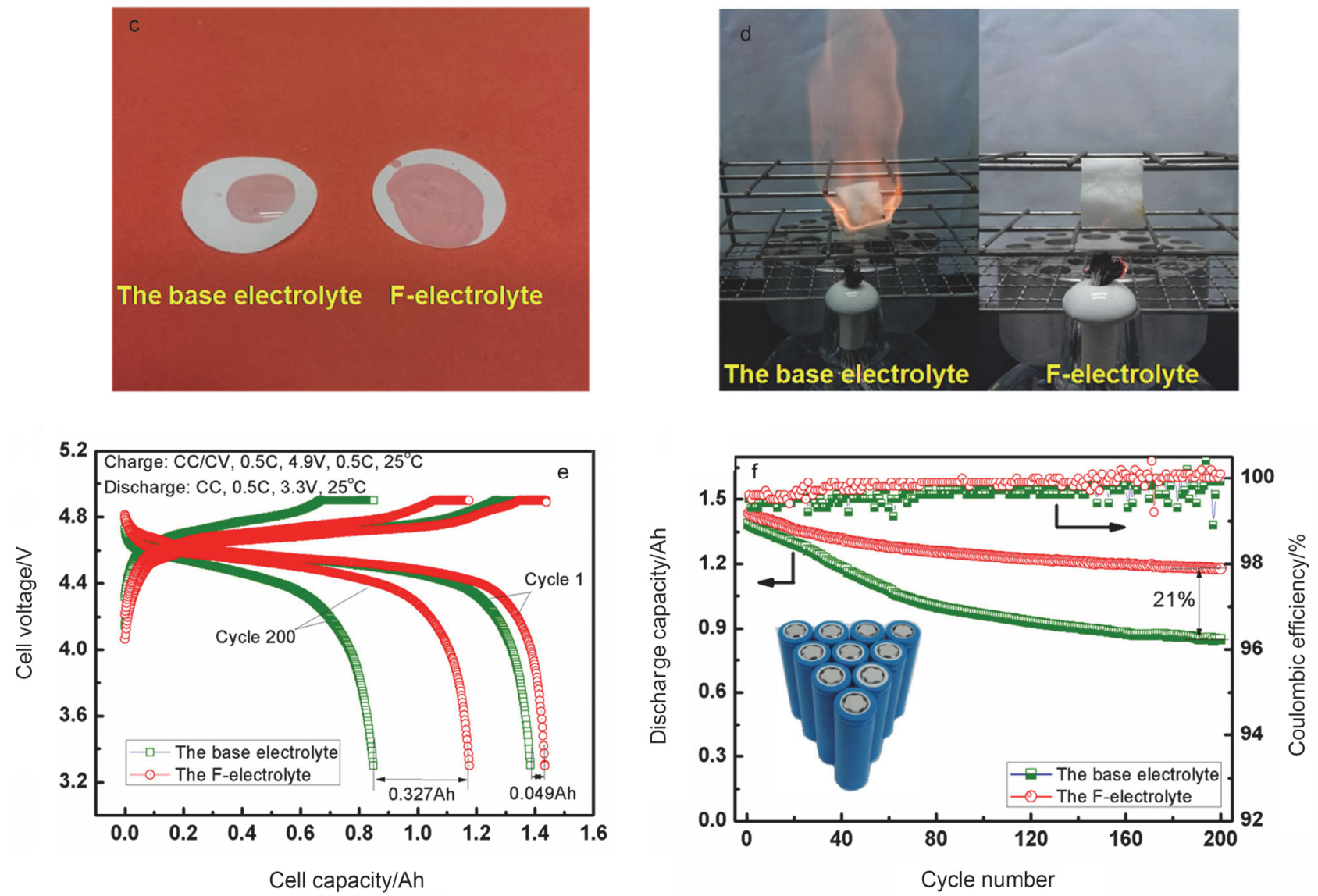

图 4 (a) 1,1,1,3,3,3-六氟异丙基甲基醚(HFPM)的结构式, (b) (d)两种电解液的物化性质比较, (e) (f) $18650 \mathrm{MCMB} / \mathrm{LiNi}_{0.5} \mathrm{Mn}_{1.5} \mathrm{O}_{4}$ 实际电池的室 温电化学性能比较 ${ }^{[72]}$

Figure 4 (a) The chemical structure of HFPM ether and (b) $\sim(d)$ the physico-chemical properties of the two electrolytes. (e) Voltage profiles for the charge-discharge cycles and (f) cycling performance according to the CC-CV protocol of the $18650 \mathrm{MCMB} / \mathrm{LiNi}{ }_{0.5} \mathrm{Mn}_{1.5} \mathrm{O}_{4}$ battery within the voltage range of $3.3 \sim 4.9 \mathrm{~V}$ at the $0.5 \mathrm{C}$ rate $^{[72]}$ 


\section{1 阻燃添加剂}

目前主要研究的阻燃添加剂为含磷的(亚)磷酸酯, 其阻燃机理为气相自由基湮灭和热阻隔机理. 当温度较 高时，电解液中的阻然剂分子气化生成含磷自由基，该 自由基可以捕获电解液中溶剂生成的氧自由基和氢氧 自由基, 生成磷酸, 最终形成热阻滞层, 覆盖在电解液 的表面, 从而阻止了电解液的燃烧 ${ }^{[80]}$. 研究表明, 一些

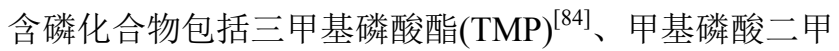
酯(DMMP) ${ }^{[85]}$ 和三苯基磷酸酯(TPPi $)^{[86]}$ 能够明显降低电 解液的可燃性, 但这些磷酸酯类化合物容易在石墨负极 共嵌、还原分解, 导致电池的循环性能差, 从而影响其 实际大规模应用. 另一些含磷化合物如六甲氧基环三磷

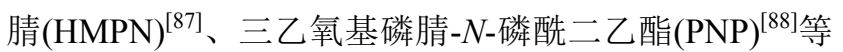
磷腈类化合物虽然与石墨类碳负极稳定性好, 但它们的 阻然效率相对较低. 为了兼具高阻燃效率和兼容性, 研 究者们合成了一些氟代的含磷化合物, 包括二(2,2,2-三 氟乙基)甲基磷酸酯(TFMP) ${ }^{[89]}$ 、2,2,2-三氟乙基二乙基磷 酸酯(TDP) ${ }^{[90,91]}$ 等, 并考察了它们的实际应用效果 ${ }^{[92]}$. 结果表明, 这些含氟磷化物不仅能大大降低电解液的可 燃性, 而且与石墨负极电化学兼容性好. 究其原因在于 $\mathrm{F}$ 原子为阻燃元素, 与 $\mathrm{P}$ 具有协同效应; 同时含氟化合 物有助于在石墨负极表面形成稳定的 SEI 膜, 提高电极 的稳定性. 因此, 氟代的含磷化合物是提高锂离子电池 安全性的高效、高匹配性阻燃剂. 在大多数情况下, 为 了使电解液完全不燃, 目前报道的阻燃剂添加量不低于 $15 \%$ (质量或体积比), 具体见表 $3^{[82 ~ 95,98]}$. 但当阻然剂 的添加量高于 $15 \%$ 时, 不仅电解液的电化学性能将受到 影响, 而且阻然剂与石墨负极不兼容问题表现更明显. 因此, 寻找高效的、高兼容性的阻燃剂显得尤为重要. 最近, Xia 等 ${ }^{[95]}$ 合成了一种高效、高匹配性的阻燃剂, 五 氟乙氧基环三磷腈(PFPN), 并具体考察了该阻燃剂的阻 燃特性和电化学稳定性. 研究发现, 只需在电解液中添 加 $5 \mathrm{wt} \%$ 的 PFPN, 电解液就能变得完全不燃. 这表明,

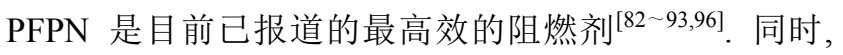
含 PFPN 的电解液能够提高 $\mathrm{LiCoO}_{2}$ 电极在截至电压为 $4.5 \mathrm{~V}$ 时的高电压循环性能. 这也表明, PFPN 既是一种 高效的阻燃剂, 又是 $\mathrm{LiCoO}_{2}$ 正极的高电压添加剂. 随 后, Feng 等 ${ }^{[97]}$ 将 PFPN 阻燃剂应用于钠离子电池, 该电 池具有高效的阻然性能的同时还表现出优异的电化学 循环性能.

\section{2 不燃或耐燃型共溶剂}

有机含氟化合物具有熔点低、闪点高的特性, 人们 将其作为电解液的共溶剂降低电解液的可燃性, 改善了 电池的安全性. Arai 等 ${ }^{[99]}$ 首先将具有高闪点的三氟丙烯 碳酸酯(TFPC) 和氯代碳酸乙烯酯 (ClEC)作为 EC 和 PC 的共溶剂来提高电池的安全性. 随后, 他们利用不燃的 含氟醚一一氟代正丁基甲基醚(MFE)制备了无闪点的
电解液 $(\mathrm{NFE})$, 该电解液的组成为 $1 \mathrm{~mol} \cdot \mathrm{L}^{-1} \mathrm{LiBETI}$ $\left[\mathrm{LiN}\left(\mathrm{SO}_{2} \mathrm{C}_{2} \mathrm{~F}_{5}\right)_{2}\right] / \mathrm{MFE}+\mathrm{EMC}(80: 20$, by vol.). 装配该 电解液的石墨 $/ \mathrm{LiCoO}_{2}$ (型号为 18650 圆柱电池)电池通 过了针刺实验, 表现出较高的安全性 ${ }^{[100,101]}$. 然而, 由于 含氟醚的含量 $\geqslant 80 \mathrm{vol} \%$ 且锂盐难溶于其中, 大剂量的 使用 MFE 降低了电解液的电导率(随着 MFE 含量从 20 $\mathrm{vol} \%$ 增加至 $80 \mathrm{vol} \%$, 相应的电解液 $1 \mathrm{~mol} \cdot \mathrm{L}^{-1} \mathrm{LiBETI} /$ $\mathrm{MFE}+\mathrm{EMC}$ 的电导率从 $2.39 \mathrm{mS} \cdot \mathrm{cm}^{-1}$ 降低至 $0.5 \mathrm{mS}$ $\left.\mathrm{cm}^{-1}\right)$. 为了解决这一问题, Naoi 等 ${ }^{[102,103]}$ 使用了支链状 含氟醚 TMMP 作为 EC 和 DEC 的共溶剂用. 结果发现, 当加入 TMMP 的含量为 $50 \mathrm{vol} \%$ 时, 电解液 $1 \mathrm{~mol} \cdot \mathrm{L}^{-1}$ LiBETI/EC +DEC+TMMP (5：45：50, by vol.)完全不 燃, 使用此电解液的电池具有较好的倍率性能以及低温 性能.<smiles>O=C1OCC(C(F)(F)F)O1</smiles>

TFPC
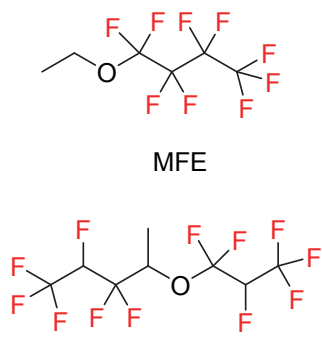

TPTP

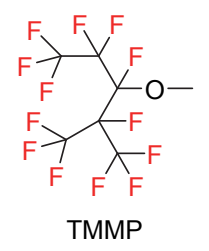

图 5 一些有机含氟化合物的结构式

Figure 5 Chemical structures of some fluorinated solvents

离子液体不仅可以应用于高电压电解液, 而且因其 本身具有几乎不挥发、不燃等特点，也常被用于制备耐 燃性电解液、降低电解液的可燃性 ${ }^{[104 ~ 106]}$, 从而提高电 池的安全性 ${ }^{[107]}$. 然而, 由于纯离子液体基电解液的粘 度较大、与隔膜等电极材料的浸润性差, 限制了锂离子 在充放电过程中的快速迁移; 且大多数的离子液体与石 墨类负极的兼容性较差, 因而, 离子液体较难作为单一 溶剂使用. 为了解决纯离子液体使用过程中产生的上述 问题，离子液体通常与碳酸酯类溶剂混合使用制备高性 能电解液. 其中, 碳酸酯类溶剂用来降低电解液的粘 度、在石墨负极表面形成稳定的 SEI 膜, 离子液体作为 共溶剂发挥其不燃、难燃的特性 ${ }^{[108 ~ 110]}$. 目前报道较多 的离子液体主要是吡咯型离子液体. 研究发现, 通过在 吡咯环上引入 $\mathrm{C}-\mathrm{O}$ 醚键或平面结构的 $\mathrm{C}=\mathrm{N}$ 双键可以 降低离子液体的粘度、提高其自身的离子电导率 ${ }^{[111,112]}$. 与不含醚键的甲丙基吡咯烷阳离子型离子液体相比(2 $\mathrm{mS} \cdot \mathrm{cm}^{-1}$ at $20{ }^{\circ} \mathrm{C}$ ), 含 $\mathrm{C}-\mathrm{O}$ 键的 $N$-甲氧基乙基- $N$-甲基 吡咯烷-双三氟甲烷磺酰亚胺阴离子 $\left(\mathrm{PYR}_{1,2 \mathrm{O} 1} \mathrm{TFSI}\right.$, 如 图 6)具有较小的粘度和较大的电导率 $\left(2.9 \mathrm{mS} \cdot \mathrm{cm}^{-1}\right.$ at $20{ }^{\circ} \mathrm{C}$ ); 同时, 兼具 $\mathrm{C}=\mathrm{N}$ 双键、醚键的 $N$-乙基-2-甲氧 基吡咯烷-双氟磺酰亚胺阴离子 [E(OMe)Pyrl-FSI]的室 
表 3 已报道的阻燃剂性能比较 ${ }^{[82 \sim 95,98]}$

Table 3 Numerous flame retardant additives tested in most of the standard Li-ion electrolytes with mixed results as indicated in as follows ${ }^{[82 \sim 95,98]}$

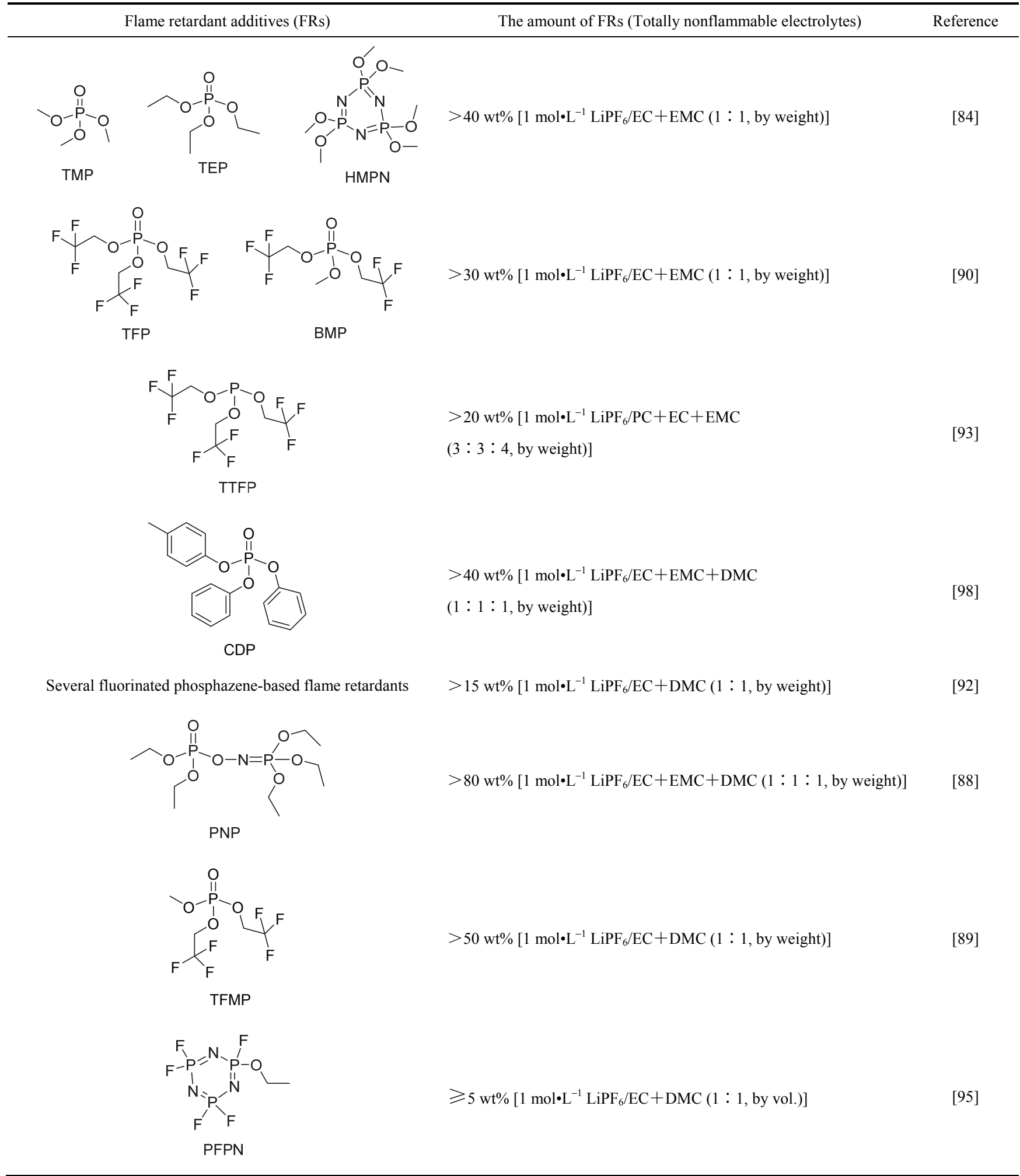

温电导率可提高至 $13 \mathrm{mS} \bullet \mathrm{cm}^{-1}$. 随后, Quinzeni 和 Kim 等 ${ }^{[113,114]}$ 系统考察了上述两种离子液体(IL)与碳酸酯类 溶剂(OCs)混合电解液体系的物化性质及在实际电池中 的电化学性能, 如图 7 所示. 研究发现, 当离子液体 IL 在混合电解液溶剂中的含量为 $60 \mathrm{wt} \%$ or vol $\%$ 时, 混合
电解液完全不燃、并显示出最佳的综合性能，其离子电 导率达到最高 $\left(9 \mathrm{mS} \cdot \mathrm{cm}^{-1}\right.$ at $\left.25{ }^{\circ} \mathrm{C}\right)$, 且粘度小、电化学 窗口宽 $(4.5 \mathrm{~V})$ 并与金属 $\mathrm{Li}$ 形成稳定界面. $\mathrm{LiFePO}_{4}$ 电池 在该混合电解液中具有优异的循环稳定性，在 $1 \mathrm{C}$ 倍率 下循环 250 周, 容量保持率为 $93 \%^{[113]}$. 
关于耐燃性电解液的研究, 目前主要的研究内容是 将具有阻燃或不燃特性的含磷系化合物、含氟溶剂、离 子液体等溶剂作为电解液的添加剂或共溶剂使用, 以降 低电解液的可燃性. 然而, 为了使电解液达到完全不燃 的程度往往需要加入足够的量, 由于这些溶剂本身存在 一些问题, 如粘度大、与石墨类负极不兼容、溶解盐的 能力小、合成成本较高等, 往往导致电解液的综合性能 下降. 因此, 设计合成具有难燃、离子电导高、电化学 性质稳定的有机溶剂是制备耐燃性、高安全性电解液的 关键所在.
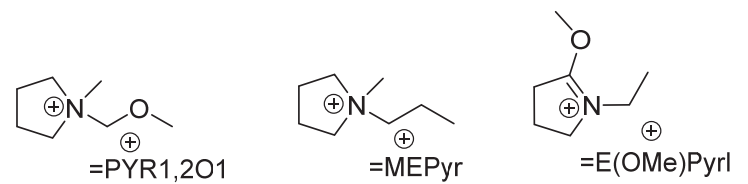

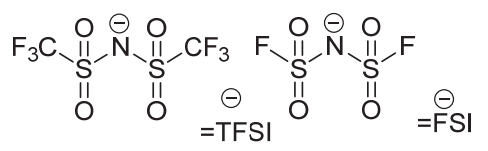

图 6 一些离子液体阳离子和阴离子的结构式

Figure 6 Chemical structures of some cation and anion in ionic liquids

\section{4 高浓度电解液}

自 1990 年锂离子电池商品化以来, 人们普遍认为: 锂离子电池电解液中有两个必不可少的组分, 即 $\mathrm{LiPF}_{6}$ 锂盐和碳酸乙烯酯(EC)溶剂, 前者兼顾了电解液对高电 导率、高电化学稳定性及高电位下钝化正极集流体 $\mathrm{Al}$ 䈃等要求, 且鉴于成本和性能的综合考虑, 锂盐的浓度 多为 $0.8 \sim 1.5 \mathrm{~mol} \cdot \mathrm{L}^{-1}$ 之间; 后者与石墨类负极兼容性 好, 能在石墨类碳负极表面形成稳定、有效的 SEI 膜, 从 而大大稳定了电池的循环性能.

近三四年, 研究者们开发了一类电解液新体系—— “超高浓度电解液”, 该类电解液中锂盐的浓度高达 4 $\mathrm{mol} \cdot \mathrm{L}^{-1}$, 远远高于普通电解液锂盐的浓度 $1 \mathrm{~mol} \cdot \mathrm{L}^{-1}$. 由于超高的锂盐含量使电解液中所有溶剂基本都与 $\mathrm{Li}^{+}$ 直接配位, 而在普通电解液中溶剂则是以游离态和溶剂 化的状态存在. 这使得超高浓度电解液具有一些特殊的 性能，包括高氧化稳定性、高还原稳定性、高热稳定性、 耐燃性及高电位下钝化 $\mathrm{Al}$ 正极集流体等 ${ }^{[115,116]}$. 研究发 现, 高浓度电解液如 $4.45 \mathrm{~mol} \cdot \mathrm{L}^{-1} \quad \mathrm{LiPF}_{6} / \mathrm{PC}$ 及 10.8 $\mathrm{mol} \cdot \mathrm{L}^{-1} \mathrm{LiN}\left(\mathrm{SO}_{2} \mathrm{~F}_{2}\right)_{2} / \mathrm{DMC}(\mathrm{LiFSA} / \mathrm{DMC}$, molar ratio= $1: 1.1$ )具有正极稳定性高(达 $5.5 \mathrm{~V} \mathrm{vs.} \mathrm{Li}^{+} / \mathrm{Li}$ )、耐燃、 热稳定性高等特点(如图 8 所示 $)^{[117,118]}$. $5 \mathrm{~V}$ 级高电压锂 离子电池一石墨/ $\mathrm{LiNi}_{0.5} \mathrm{Mn}_{1.5} \mathrm{O}_{4}$ 在高浓度 $\mathrm{LiFSA} / \mathrm{DMC}$ (1: 1.1) 电解液中 $40{ }^{\circ} \mathrm{C}$ 下 100 周后容量保持率为 $90 \%$, 远高于电池在常规电解液中的 $18 \%$, 显示出优异的高电 压循环稳定性. 同时, 锂盐为 LiTFSI 和 LiFSA 的超高浓 度电解液在高电位下能在 $\mathrm{Al}$ 正极集流体表面形成良好 的钝化膜，从而有效抑制了以 $\mathrm{N}$ 为中心原子的锂盐如 LiTFSI 和 LiFSA 等对正极集流体 $\mathrm{Al}$ 䇴的腐蚀作 用 ${ }^{[119 \sim 121]}$.

在常规电解液中, 锂盐的浓度为 $1 \mathrm{~mol} \cdot \mathrm{L}^{-1}$. 以石墨 为负极的锂离子电池必须使用 $\mathrm{EC}$ 作溶剂; 而 $\mathrm{EC}$ 以外 的其它溶剂如碳酸丙烯酯(PC)、醚类如二甲醚(DME)、 腈类如乙腈( $\mathrm{AN})$ 以及亚砜类如二甲基亚砜(DMSO)等不 能单独作电解液溶剂用, 其原因在于它们均不能在石墨 表面形成稳定的 SEI 膜、且共嵌入石墨层间，破坏石墨 的层间结构, 从而引起石墨负极电化学性能的恶 化 ${ }^{[122 ~ 125]}$. 然而, 当其电解液中锂盐含量超高, 制备成 超高浓度电解液时, 锂盐的阴离子优先于溶剂分子在石 墨表面还原分解, 形成具有高离子电导的 SEI 膜, 有效 抑制了溶剂分子的共嵌入，从而大大提高了石墨负极的 循环稳定性及倍率性能，如图 9 所示 ${ }^{[126,127]}$. 同样地，由 于超高浓度电解液与金属锂之间界面稳定性的提高，大 大改善了金属锂的可逆溶解一沉积反应，减少了金属锂

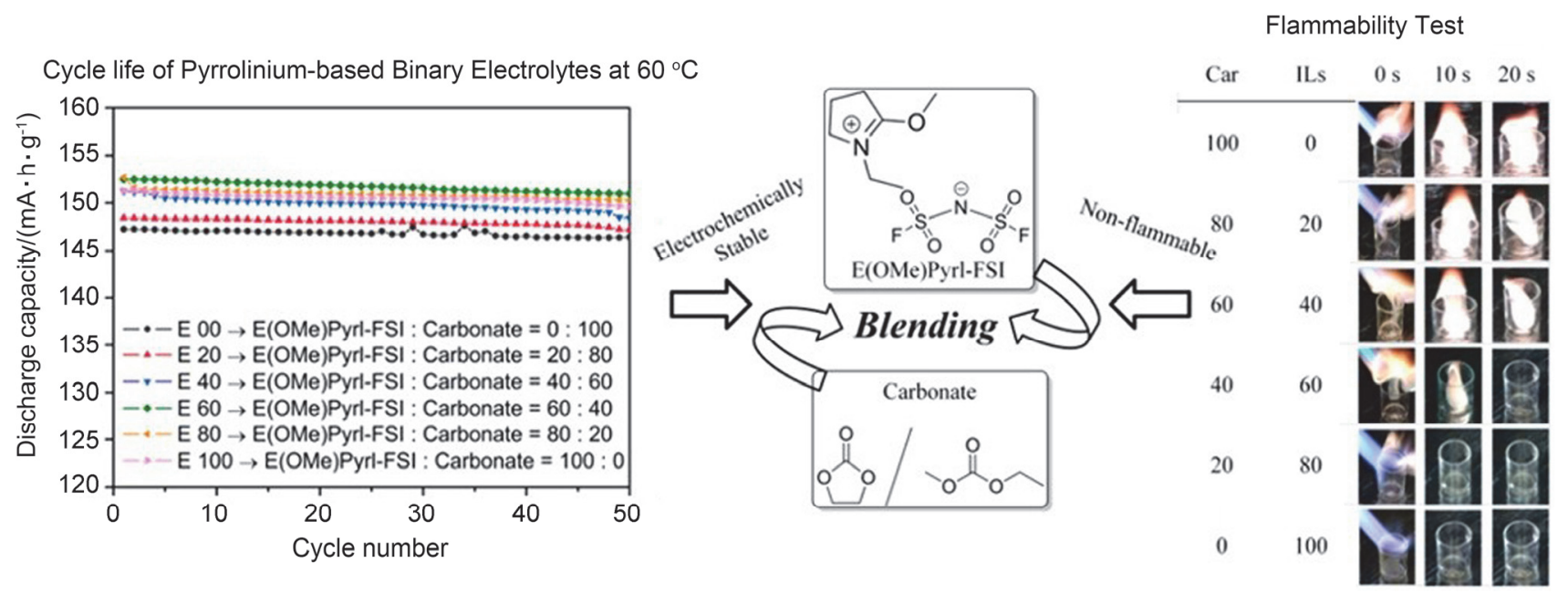

图 7 离子液体一碳酸酯类溶剂二元混合电解液的可燃性测试及其在电池 $\mathrm{Li} / \mathrm{LiFePO}_{4}$ 中的高温 $60{ }^{\circ} \mathrm{C}$ 循环性能 ${ }^{[114]}$

Figure 7 Left: cycle performance of the $\mathrm{Li} / \mathrm{LiFePO}_{4}$ cells in six different electrolytes at $25{ }^{\circ} \mathrm{C}$; E 00 (black), E 20 (red), E 40 (blue), E 60 (green), E 80 (orange), and E 100 (pink). Right: images of the flammability tests of E 00, E 20, E 40, E 60, E 80, E $100^{[114]}$ 

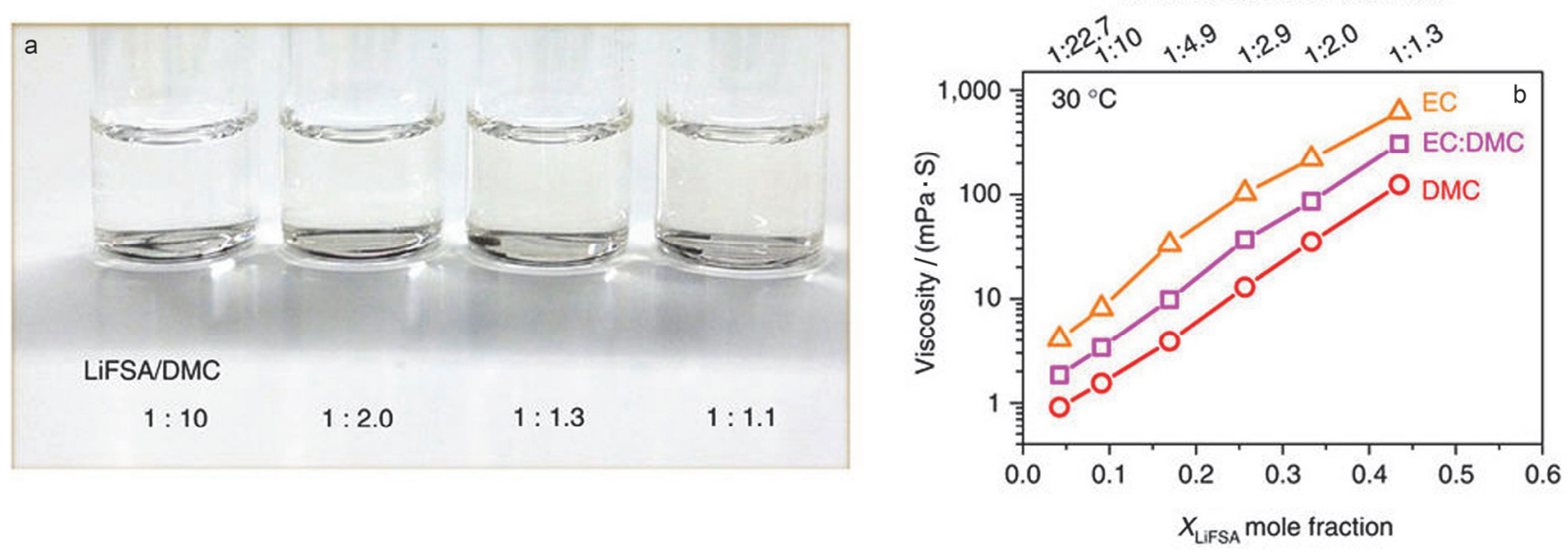

LiFSA-to-solvent molar ratio
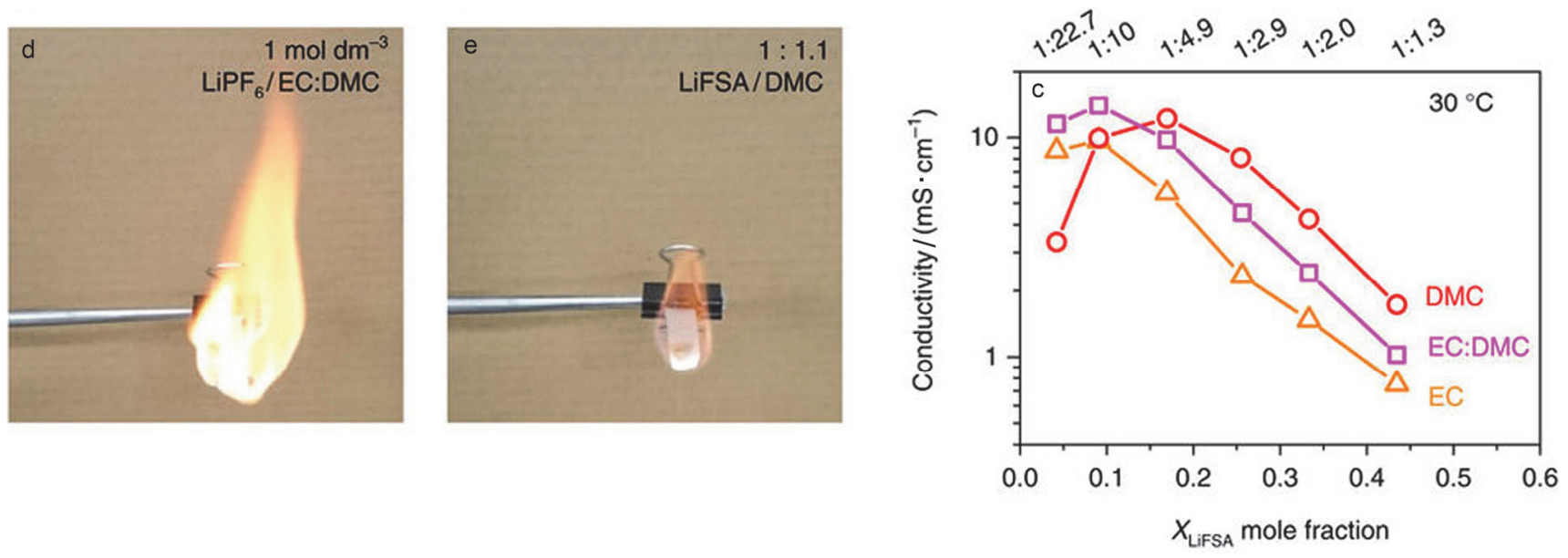

图 8 不同物质的量比的 LiFSA/DMC 电解液的物化性质比较 ${ }^{[118]}$

Figure 8 Physicochemical properties dependent on solution concentration ${ }^{[118]}$

表面枝晶的形成 ${ }^{[128 \sim 130]}$. Qian 等 ${ }^{[129]}$ 报道了金属 $\mathrm{Li}$ 在 4.0 $\mathrm{mol} \cdot \mathrm{L}^{-1} \mathrm{LiFSA} / \mathrm{DME}$ 高浓度电解液中的优异的电化学 性能. 他们发现, $\mathrm{Li} / \mathrm{Li}$ 电池在该电解液中以 $10 \mathrm{~mA} \cdot \mathrm{cm}^{-2}$ 电流密度循环 6000 周库仑效率高达 $99.1 \%$, 显示出优异 的循环稳定性.

相比于常规电解液, 高浓度电解液因溶液中溶剂锂盐间强的相互作用使得其具有普通电解液所无法比 拟的诸多特殊性能, 如氧化还原性高、耐燃等, 但这些 性能的提高是以电解液粘度增加、电导率降低及成本增 加为代价的 (表 4 对上述的锂离子电池三类电解液基本 性能进行了比较). 然而, 我们也应当看到, 高浓度电解 液打破了电解液制备过程中的一些现有束缚, 为高电压 石墨 $/ \mathrm{LiNi}_{0.5} \mathrm{Mn}_{1.5} \mathrm{O}_{4}$ 锂离子电池、以 $\mathrm{Li}$ 金属为负极的高 容量 $\mathrm{Li} / \mathrm{S}$ 或 $\mathrm{Li}-$ 空气电池以及钠离子电池的电解液设计 和开发提供新的设计思路.

\section{5 总结与展望}

自锂离子电池商品化至今, 锂离子电池正、负极材 料的发展迅速, 正极材料从 $\mathrm{LiCoO}_{2}$ 到三元材料、高电压
$\mathrm{LiNi}_{0.5} \mathrm{Mn}_{1.5} \mathrm{O}_{4}$ 、高容量富锂锰基正极材料等, 负极材料 则从石墨类碳负极发展到高容量硅基负极材料, 高容量 电极材料的蓬勃发展必然要求相应的电解液体系、电池 工艺等的提升. 然而, 相对于锂离子电池电极材料的蓬 勃发展, 新型电解液的研发相对滞后. 目前应用成熟的 电解液体系仍然是常规碳酸酯类溶剂体系, 该电解液体 系存在正极稳定性差、闪点低、易燃烧等问题.一方面， 为了提高电解液在正极的高电位下稳定性，可以通过在 电解液加入添加剂, 然而其耐受电压上限仍只有 $4.5 \mathrm{~V}$, 进一步提高电解液的抗氧化性必须要开发新型的电解 液体系. 从目前的研究情况看, 氟代碳酸酯类和离子液 体是高电压电解液溶剂的最佳选择，同时需要加入合适 的添加剂改善其与负极界面的稳定性. 另一方面, 为了 降低常规碳酸酯的电解液的可燃性, 单纯的通过添加阻 燃剂很难从根本上解决其易燃问题, 亟待开发耐燃性的 电解液用溶剂. 在实际应用中, 当电池处于热失控状态 时, 电池内部温度和压力均很高, 安全阀开启或外壳破 裂时，阻燃性电解液会以极快的速度喷出，足以使其中 低闪点的组分被点燃，导致电池燃烧. 由此可见，含有 

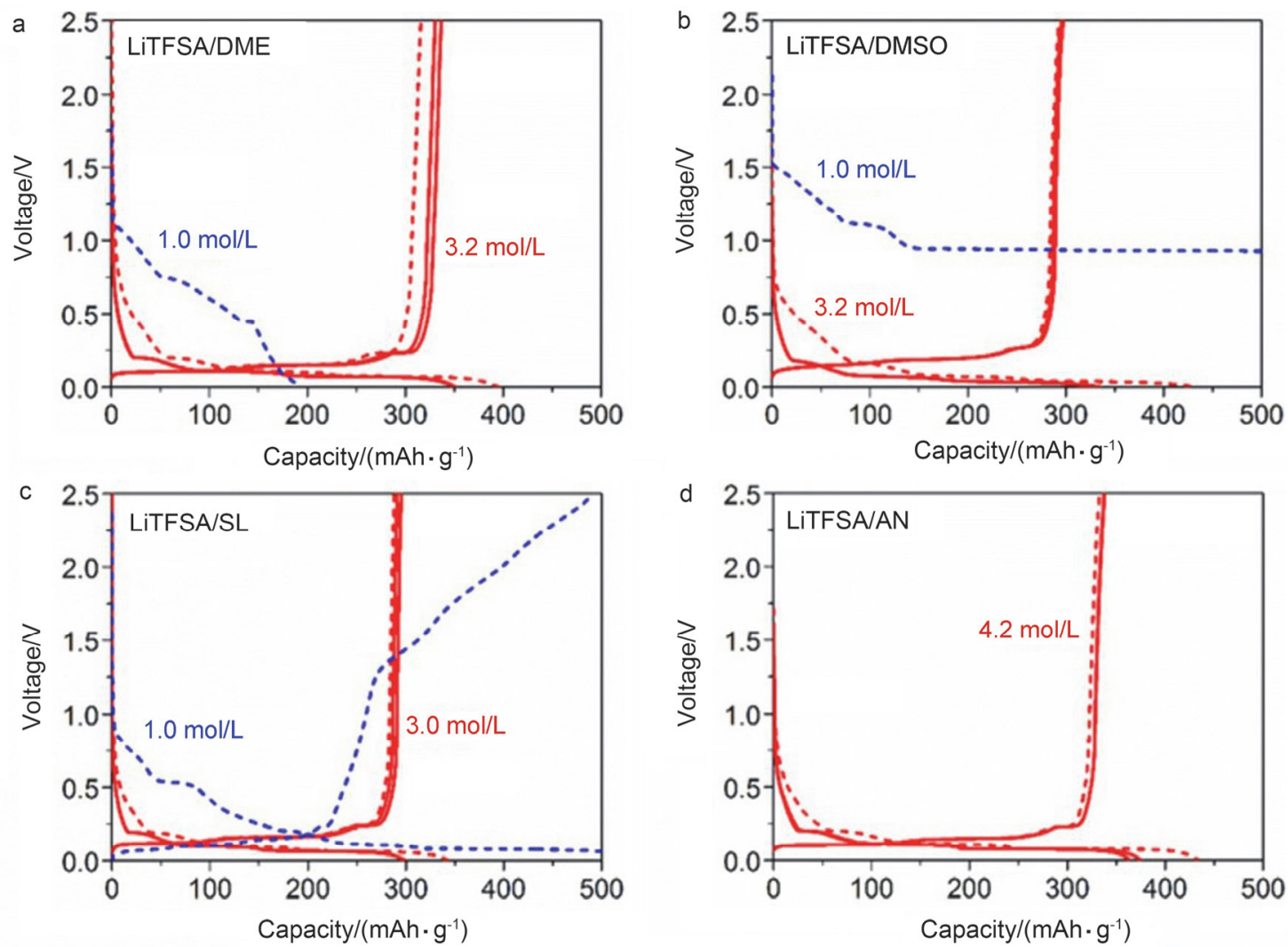

图 9 电池 $\mathrm{Li} /$ 石墨在超高浓度电解液及相应的普通电解液中的充放电曲线对比 ${ }^{[115]}$

Figure 9 Charge-discharge curves of natural graphite/Li metal half cells with dilute and concentrated LiTFSA electrolytes of (a) DME, (b) DMSO, (c) $\mathrm{SL}$, and (d) AN at $\mathrm{C} / 10$ rate and $25{ }^{\circ} \mathrm{C}$. All the highly concentrated electrolytes correspond to the solvent/Li salt molar ratio of $c a$. 2. The charge-discharge test with a dilute LiTFSA/AN electrolyte was not possible because of high reactivity of AN solvent with Li metal ${ }^{[115]}$

表 4 锂离子电池三类电解液的基本性能比较

Table 4 Properties of different types of electrolyte used in lithium ion batteries

\begin{tabular}{|c|c|c|c|c|c|c|}
\hline Electrolyte & Typical example & Viscosity/(mPa $\bullet$ s) & Conductivity $/\left(\mathrm{mS} \cdot \mathrm{cm}^{-1}\right)$ & $\begin{array}{l}\text { Electrochemical } \\
\text { window/V }\end{array}$ & Flammability & Ref. \\
\hline \multirow{7}{*}{$\begin{array}{l}\text { High voltage } \\
\text { electrolytes }\end{array}$} & $\begin{array}{l}1 \mathrm{~mol} \cdot \mathrm{L}^{-1} \mathrm{LiPF}_{6} / \mathrm{EC}+\mathrm{DMC}(1: 1 \mathrm{in} \\
\mathrm{wt} \%)- \text { Sebaconitrile }(50: 50 \text {, by vol. })\end{array}$ & - & $4.4,25{ }^{\circ} \mathrm{C}$ & $\begin{array}{l}\sim 7.5 \text {, glassy carbon } \\
\text { electrode }\end{array}$ & - & [39] \\
\hline & $\begin{array}{l}1 \mathrm{~mol} \cdot \mathrm{L}^{-1} \mathrm{LiBF}_{4} / \mathrm{EC}-\mathrm{DMC}-\text { Sebaconitrile } \\
(25: 25: 50 \text {, by vol. })\end{array}$ & - & $1.8,25{ }^{\circ} \mathrm{C}$ & $\begin{array}{l}0.4 \sim 7.0, \text { glassy carbon } \\
\text { electrode }\end{array}$ & - & {$[39,40]$} \\
\hline & $\begin{array}{l}1 \mathrm{~mol} \cdot \mathrm{L}^{-1} \text { Lithium bis(trifluoromethane } \\
\text { sulfonyl) imide (Lilm)/Ethyl methyl } \\
\text { sulfone (EMSF) }\end{array}$ & - & $6.31,25{ }^{\circ} \mathrm{C}$ & $\begin{array}{l}0 \sim 5.8, \text { Pt working } \\
\text { electrode }\end{array}$ & - & {$[45]$} \\
\hline & $\begin{array}{l}1.0 \mathrm{~mol} \cdot \mathrm{L}^{-1} \mathrm{LiTFSI} / \text { ethylmethoxyethyl } \\
\text { sulfone }(\mathrm{EMES})\end{array}$ & - & $1.41,25{ }^{\circ} \mathrm{C}$ & $\begin{array}{l}0 \sim 5.6 \text {, Pt working } \\
\text { electrode }\end{array}$ & - & [47] \\
\hline & $\begin{array}{l}0.5 \mathrm{~mol} \cdot \mathrm{L}^{-1} \mathrm{LiTFSI} / \mathrm{PP}_{14}-\mathrm{TFSI}-\mathrm{TMS} \\
(60: 40 \text {, by wt. })\end{array}$ & - & $2.04,25^{\circ} \mathrm{C}$ & $\begin{array}{l}0 \sim 5.7, \text { Pt working } \\
\text { electrode }\end{array}$ & Non-flammability & {$[65]$} \\
\hline & $\begin{array}{l}1 \mathrm{~mol} \cdot \mathrm{L}^{-1} \mathrm{LiPF}_{6} / \mathrm{FEC}-\mathrm{TFTFEP} \\
(1: 1, \text { by vol. })\end{array}$ & $9.44,25{ }^{\circ} \mathrm{C}$ & $3.51,25{ }^{\circ} \mathrm{C}$ & - & - & [67] \\
\hline & $\begin{array}{l}1 \mathrm{~mol} \cdot \mathrm{L}^{-1} \\
\mathrm{LiPF}_{6} / \text { FEC-DMC-EMC-HFPM } \\
(2: 3: 1: 4, \text { by vol. })\end{array}$ & - & $8.57,30{ }^{\circ} \mathrm{C}$ & $\begin{array}{l}0 \sim 5.5 \text {, Pt working } \\
\text { electrode }\end{array}$ & Non-flammability & {$[72]$} \\
\hline
\end{tabular}




\begin{tabular}{|c|c|c|c|c|c|c|}
\hline Electrolyte & Typical example & Viscosity/(mPa•s) & Conductivity $/\left(\mathrm{mS} \cdot \mathrm{cm}^{-1}\right)$ & $\begin{array}{l}\text { Electrochemical } \\
\text { window } / \mathrm{V}\end{array}$ & Flammability & Ref. \\
\hline \multirow{5}{*}{$\begin{array}{l}\text { Flame } \\
\text { retardant } \\
\text { electrolytes }\end{array}$} & $1 \mathrm{~mol} \cdot \mathrm{L}^{-1} \mathrm{LiPF}_{6} / \mathrm{ClEC}-\mathrm{TFPC}$ & - & $3 \sim 4,25{ }^{\circ} \mathrm{C}$ & - & - & $\overline{[99]}$ \\
\hline & $\begin{array}{l}1 \mathrm{~mol} \cdot \mathrm{L}^{-1} \text { LiBETI-MFE/EMC }(80: 20 \text {, } \\
\text { by vol.) }\end{array}$ & - & $0.56,25{ }^{\circ} \mathrm{C}$ & - & No flash point & [100] \\
\hline & $\begin{array}{l}1 \mathrm{~mol} \cdot \mathrm{L}^{-1} \mathrm{LiBETI} / \mathrm{EC}+\mathrm{DEC}+\mathrm{TMMP} \\
(5: 45: 50 \text {, by vol. })\end{array}$ & - & $1.0,20{ }^{\circ} \mathrm{C}$ & $\begin{array}{l}0 \sim 5.6, \text { Pt working } \\
\text { electrode }\end{array}$ & Non-flammability & {$[102]$} \\
\hline & $\begin{array}{l}0.5 \mathrm{~mol} \cdot \mathrm{L}^{-1} \mathrm{LiTFSI} / \mathrm{EC}-\mathrm{DEC} \\
\mathrm{PYR}_{1,2} \mathrm{O}_{1} \mathrm{TFSI} \text {, by vol. }\end{array}$ & $25,20{ }^{\circ} \mathrm{C}$ & $7.0,20{ }^{\circ} \mathrm{C}$ & $\begin{array}{l}0 \sim 4.5, \text { Ni working } \\
\text { electrode }\end{array}$ & Non-flammability & [113] \\
\hline & $\begin{array}{l}1 \mathrm{~mol} \cdot \mathrm{L}^{-1} \mathrm{LiPF}_{6} / \text { EC-EMC }(3: 7, \text { by vol. }) \\
+1 \mathrm{~mol} \cdot \mathrm{L}^{-1} \mathrm{LiTFSI} / \mathrm{E}(\mathrm{OMe}) \text { Pyrl-FSI } \\
(20: 80, \text { by wt. } \%)\end{array}$ & $25,20{ }^{\circ} \mathrm{C}$ & $6.9,20{ }^{\circ} \mathrm{C}$ & $\begin{array}{l}1.2 \sim 4.5 \text {, glassy carbon } \\
\text { electrode }\end{array}$ & Non-flammability & [114] \\
\hline \multirow{6}{*}{$\begin{array}{l}\text { Highly } \\
\text { concentrated } \\
\text { electrolytes }\end{array}$} & $4.27 \mathrm{~mol} \cdot \mathrm{kg}^{-1} / \mathrm{PC}$ & $706.5,30{ }^{\circ} \mathrm{C}$ & $0.403,30{ }^{\circ} \mathrm{C}$ & $\begin{array}{l}\sim 5.0, \text { Pt working } \\
\text { electrode }\end{array}$ & - & [117] \\
\hline & LiFSA/DMC $1: 1.1$ molar ratio & $238.9,30{ }^{\circ} \mathrm{C}$ & $1.1,30{ }^{\circ} \mathrm{C}$ & $>5.5 \mathrm{~V}, \mathrm{Al}$ electrode & Not burn & [118] \\
\hline & LiFSA/AN $1: 1.5$ molar ratio & $153,30{ }^{\circ} \mathrm{C}$ & $3.3,30{ }^{\circ} \mathrm{C}$ & $>4.5 \mathrm{~V}, \mathrm{Al}$ electrode & - & [121] \\
\hline & LiFSA/DME $1: 1.9$ molar ratio & $25.1,30{ }^{\circ} \mathrm{C}$ & $7.2,30{ }^{\circ} \mathrm{C}$ & - & - & [126] \\
\hline & $\begin{array}{l}7 \mathrm{~mol} \cdot \mathrm{L}^{-1} \text { LiTFSI/DOL-DME } \\
(1: 1 \text {, by vol. })\end{array}$ & $72,25{ }^{\circ} \mathrm{C}$ & $0.814,25{ }^{\circ} \mathrm{C}$ & - & - & [128] \\
\hline & $5 \mathrm{~mol} \cdot \mathrm{L}^{-1} \mathrm{LiTFSI} / \mathrm{DME}$ & - & $1.7,25^{\circ} \mathrm{C}$ & $\begin{array}{l}0 \sim 5.0, \text { Pt working } \\
\text { electrode }\end{array}$ & - & [129] \\
\hline
\end{tabular}

阻燃剂的电解液并不能从根本上消除电池燃烧事故的 发生. 因此, 开发高闪点溶剂的电解液才可能从根本上 解决电池发生燃烧的安全隐患. 从目前的研究进展来 看, 离子液体因具有不挥发性、不燃、温度范围宽, 且 溶解能力强等优点或将成为不燃电解液的最佳选择. 因 此, 未来几年, 随着信息技术的发展以及新能源汽车的 进一步推广, 开发新型抗氧化性溶剂、提高电极/电解液 界面稳定性, 发展高电压、耐燃性电解液已成为目前电 解液行业研究的重点. 此外, 超高浓度电解液表现出诸 多不同于常规浓度电解液的优势, 其研究的兴起必然为 电解液的设计提供了新方向.

\section{作者简介}

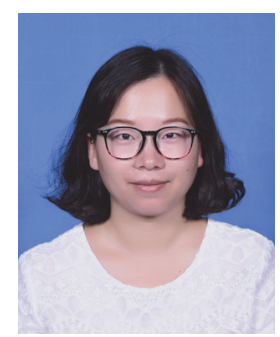

夏兰, 宁波诺丁汉大学副研究员. 2013 年在武汉大学化学 与分子科学学院获得博士学位, 导师艾新平教授. 主要研究
领域为高性能电解液、锂离子电池安全性技术. 作为负责人主 持国家自然科学基金青年基金、中国博士后科学基金面上(一 等)资助、宁波市自然科学基金, 作为合作单位负责人主持省 属科研院所专项项目 1 项.

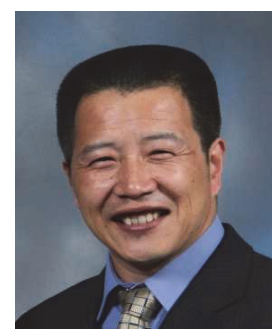

陈政(George Zheng Chen), 英国诺丁汉大学化学与环境 工程系(英、中校区)正教授、可持续能源技术研究中心主任. 1992 年在伦敦大学帝国理工医学院获博士学位. 2000 年应聘 于教育部长江学者奖励计划特聘教授. 2002 年获得自然科学 基金会杰出青年基金. 2015 年入选浙江省千人计划. 长期从事 电化学基础和应用研究, 涉及领域包括材料、能源和环境. 迄 今，陈政教授已在 Nature, Angew. Chem. Int. Edi., Adv. Mater., Energy Environ. Sci., Chem. Commun., J. Am. Chem. Soc. 等有 同行评议的学术期刊上发表原始研究和综述论文 190 余篇; 获准国内外专利 19 项; 发表学术会议报告 200 余篇; 研究论 文的总 SCI 引用次数超过 8000 次, 其中有 19 篇论文的单篇 SCI 引用次数超过 100 次. 据 SCI 和 Google Scholar 统计, 陈 政的 h-index 分别为 52 和 60 . 


\section{References}

[1] Aurbach, D.; Talyosef, Y.; Markovsky, B.; Markevich, E.; Zinigrad, E.; Asraf, L.; Gnanaraj, J. S.; Kim, H. J. Electrochim. Acta 2004, 50, 247.

[2] Markovsky, B.; Amalraj, F.; Gottlieb, H. E.; Gofer, Y.; Martha, S. K.; Aurbach, D. J. Electrochem. Soc. 2010, 157, A423.

[3] Erickson, E. M.; Markevich, E.; Salitra, G.; Sharon, D.; Hirshberg, D.; de la Llave, E.; Shterenberg, I.; Rozenman, A.; Frimer, A.; Aurbach, D. J. Electrochem. Soc. 2015, 162, A2424.

[4] Xu, K. Chem. Rev. 2004, 104, 4303.

[5] Hu, M.; Pang, X.; Zhou, Z. J. Power Sources 2013, 237, 229.

[6] Xu, K. Chem. Rev. 2014, 114, 11503.

[7] Wan, Y.; Zheng, Q.-J.; Lin, D.-M. Acta Chim. Sinica 2014, 72, 537. (万洋, 郑养佶, 赁敦敏, 化学学报, 2014, 72, 537.)

[8] Xing, L.; Li, W.; Wang, C.; Gu, F.; Xu, M.; Tan, C.; Yi, J. J. Phys. Chem. B 2009, 113, 16596.

[9] Yang, L.; Ravdel, B.; Lucht, B. L. Electrochem. Solid-State Lett. 2010, 13, A95.

[10] Kim, J.-H.; Pieczonka, N. P. W.; Li, Z.; Wu, Y.; Harris, S.; Powell, B. R. Electrochim. Acta 2013, 90, 556.

[11] Pieczonka, N. P. W.; Liu, Z.; Lu, P.; Olson, K. L.; Moote, J.; Powell, B. R.; Kim, J.-H. J. Phys. Chem. C 2013, 117, 15947.

[12] Yao, X. L.; Xie, S.; Chen, C. H.; Wang, Q. S.; Sun, J. H.; Li, Y. L.; Lu, S. X. J. Power Sources 2005, 144, 170.

[13] Zhang, H. P.; Xia, Q.; Wang, B.; Yang, L. C.; Wu, Y. P.; Sun, D. L.; Gan, C. L.; Luo, H. J.; Bebeda, A. W.; Ree, T. v. Electrochem. Commun. 2009, 11, 526.

[14] Hyung, Y. E.; Vissers, D. R.; Amine, K. J. Power Sources 2003, $119-121,383$

[15] Mandal, B. K.; Padhi, A. K.; Shi, Z.; Chakraborty, S.; Filler, R. J. Power Sources 2006, 161, 1341.

[16] Shim, E.-G.; Nam, T.-H.; Kim, J.-G.; Kim, H.-S.; Moon, S.-I. J. Power Sources 2007, 172, 901.

[17] Ren, Y.; Wen, Y.; Lian, F.; Qiu, W.-H. Chemistry 2015, 78, 107. (任 岩, 文炎, 连芳, 丑卫华, 化学通报, 2015, 78, 107.)

[18] Dalavi, S.; Xu, M.; Knight, B.; Lucht, B. L. Electrochem. Solid-State Lett. 2011, 15, A28.

[19] Yang, L.; Markmaitree, T.; Lucht, B. L. J. Power Sources 2011, 196, 2251.

[20] Hu, M.; Wei, J.; Xing, L.; Zhou, Z. J. Appl. Electrochem. 2012, 42, 291.

[21] Li, Z. D.; Zhang, Y. C.; Xiang, H. F.; Ma, X. H.; Yuan, Q. F.; Wang, Q. S.; Chen, C. H. J. Power Sources 2013, 240, 471.

[22] von Cresce, A.; Xu, K. J. Electrochem. Soc. 2011, 158, A337.

[23] von Cresce, A.; Xu, K. ECS Transactions 2012, 41, 17.

[24] Rong, H.; Xu, M.; Xing, L.; Li, W. J. Power Sources 2014, 261, 148.

[25] Song, Y.-M.; Han, J.-G.; Park, S.; Lee, K. T.; Choi, N.-S. J. Mater. Chem. A 2014, 2, 9506

[26] Yan, G.; Li, X.; Wang, Z.; Guo, H.; Wang, C. J. Power Sources 2014, $248,1306$.

[27] Zhang, J.; Wang, J.; Yang, J.; NuLi, Y. Electrochim. Acta 2014, 117, 99.

[28] Yan, G.; Li, X.; Wang, Z.; Guo, H.; Xiong, X. J. Power Sources 2014, 263, 231.

[29] Felix, F.; Cheng, J.-H.; Hy, S.; Rick, J.; Wang, F. M.; Hwang, B.-J. J. Phys. Chem. C 2013, 117(44), 22619.

[30] Lee, H.; Choi, S.; Choi, S.; Kim, H.-J.; Choi, Y.; Yoon, S.; Cho, J.-J. Electrochem. Commun. 2007, 9, 801.

[31] Tarnopolskiy, V.; Kalhoff, J.; Nádherná, M.; Bresser, D.; Picard, L.; Fabre, F.; Rey, M.; Passerini, S. J. Power Sources 2013, 236, 39.

[32] Bouayad, H.; Wang, Z.; Dupré, N.; Dedryvère, R.; Foix, D.; Franger, S.; Martin, J.-F.; Boutafa, L.; Patoux, S.; Gonbeau, D.; Guyomard, D. J. Phys. Chem. C 2014, 118(9), 4634.

[33] Pieczonka, N. P. W.; Yang, L.; Balogh, M. P.; Powell, B. R.; Chemelewski, K. R.; Manthiram, A.; Krachkovskiy, S. A.; Goward, G. R.; Liu, M.; Kim, J.-H. J. Phys. Chem. C 2013, 117(44), 22603.

[34] Xu, K.; Zhang, S.; Jow, T. R. Electrochem. Solid-State Lett. 2003, 6, A117.

[35] Wu, Q.; Lu, W.; Miranda, M.; Honaker-Schroeder, T. K.; Lakhsassi, K. Y.; Dees, D. Electrochem. Commun. 2012, 24, 78.

[36] Xu, M.; Zhou, L.; Dong, Y.; Chen, Y.; Demeaux, J.; MacIntosh, A. D.; Garsuch, A.; Lucht, B. L. Energy Environ. Sci. 2016, 9, 1308.

[37] Ue, M.; Ida, K.; Mori, S. J. Electrochem. Soc. 1994, 141, 2989.

[38] Ue, M.; Takeda, M.; Takehara, M.; Mori, S. J. Electrochem. Soc.
1997, 144, 2684

[39] Nanini-Maury, E.; Światowska, J.; Chagnes, A.; Zanna, S.; Tran-Van, P.; Marcus, P.; Cassir, M. Electrochim. Acta 2014, 115, 223.

[40] Nagahama, M.; Hasegawa, N.; Okada, S. J. Electrochem. Soc. 2010, 157, A748.

[41] Kavan L. Chem. Rev. 1997, 97, 3061.

[42] Abu-Lebdeh, Y.; Davidson, I. J. Electrochem. Soc. 2009, 156, A60.

[43] Abu-Lebdeh, Y.; Davidson, I. J. Power Sources 2009, 189, 576.

[44] Gmitter, A. J.; Plitz, I.; Amatucci, G. G. J. Electrochem. Soc. 2012, 159, A370.

[45] Xu, K.; Angell, C. A. J. Electrochem. Soc. 1998, 145, L70.

[46] Sun, X.-G.; Angell, C. A. Solid State Ionics 2004, 175, 257.

[47] Sun, X.-G.; Angell, C. A. Electrochem. Commun. 2005, 7, 261.

[48] Sun, X.; Angell, C. A. Meeting Abstracts 2008, MA2008-01, 162.

[49] Watanabe, Y.; Kinoshita, S.-i.; Wada, S.; Hoshino, K.; Morimoto, H.; Tobishima, S.-i. J. Power Sources 2008, 179, 770.

[50] Sun, X.; Angell, C. A. Electrochem. Commun. 2009, 11, 1418.

[51] Mao, L.; Li, B.; Cui, X.; Zhao, Y.; Xu, X.; Shi, X.; Li, S.; Li, F. Electrochim. Acta 2012, 79, 197.

[52] Li, C.; Zhao, Y.; Zhang, H.; Liu, J.; Jing, J.; Cui, X.; Li, S. Electrochim. Acta 2013, 104, 134.

[53] Wu, F.; Xiang, J.; Li, L.; Chen, J.; Tan, G.; Chen, R. J. Power Sources 2012, 202, 322.

[54] Wu, F.; Zhu, Q.; Li, L.; Chen, R.; Chen, S. J. Mater. Chem. A 2013 1,3659 .

[55] Abouimrane, A.; Belharouak, I.; Amine, K. Electrochem. Commun. 2009, 11, 1073 .

[56] Xing, L.; Vatamanu, J.; Borodin, O.; Smith, G. D.; Bedrov, D. J. J. Phys. Chem. C 2012, 116, 23871.

[57] Sakaebe, H.; Matsumoto, H. Electrochem. Commun. 2003, 5, 594.

[58] Matsumoto, H.; Sakaebe, H.; Tatsumi, K. J. Power Sources 2005 $146,45$.

[59] Galiński, M.; Lewandowski, A.; Stepniak, I. Electrochim. Acta 2006, $51,5567$.

[60] Armand, M.; Endres, F.; MacFarlane, D. R.; Ohno, H.; Scrosati, B. Nat. Mater. 2009, 8, 621 .

[61] Seki, S.; Serizawa, N.; Takei, K.; Miyashiro, H.; Watanabe, M. Meeting Abstracts 2011, MA2011-02, 1277.

[62] Le, M.-L.-P.; Alloin, F.; Strobel, P.; Leprêtre, J.-C.; Cointeaux, L.; Valle, C. Ionics 2012, 18, 817.

[63] Dokko, K.; Tachikawaa, N.; Yamauchia, K.; Tsuchiyaa, M.; Yamazakia, A.; Takashimaa, E.; Parka, J.-W.; Uenoa, K.; Sekib, S.; Serizawab, N.; Watanabea, M. J. Electrochem. Soc. 2013, 160, A1304.

[64] Borgel, V.; Markevich, E.; Aurbach, D.; Semrau, G.; Schmidt, M. J. Power Sources 2009, 189, 331.

[65] Xiang, J.; Wu, F.; Chen, R.; Li, L.; Yu, H. J. Power Sources 2013 $233,115$.

[66] Mun, J.; Yim, T.; Park, K.; Ryu, J. H.; Kim, Y. G.; Oh, S. M. J. Electrochem. Soc. 2011, 158, A453.

[67] Kitagawa, T.; Azuma, K.; Koh, M.; Yamauchi, A.; Kagawa, M.; Sakata, H.; Miyawaki, H.; Nakazono, A.; Arima, H.; Yamagata, M. Electrochemistry 2010, 78, 345

[68] Hu, L.; Zhang, Z.; Amine, K. Electrochem. Commun. 2013, 35, 76.

[69] Markevich, E.; Salitra, G.; Fridman, K.; Sharabi, R.; Gershinsky, G.; Garsuch, A.; Semrau, G.; Schmidt, M. A.; Aurbach, D. Langmuir 2014, 30, 7414

[70] Zhang, Z.; Hu, L.; Wu, H.; Weng, W.; Koh, M.; Redfern, P. C.; Curtiss, L. A.; Amine, K. Energy Environ. Sci. 2013, 6, 1806.

[71] Achiha, T.; Nakajima, T.; Ohzawa, Y.; Koh, M.; Yamauchi, A.; Kagawa, M.; Aoyama, H. J. Electrochem. Soc. 2010, 157, A707.

[72] Xia, L.; Xia, Y.; Wang, C.; Hu, H.; Lee, S.; Yu, Q.; Chen, H.; Liu, Z. ChemElectroChem 2015, 2, 1707.

[73] Yan, G.; Li, X.; Wang, Z.; Guo, H.; Wang, J. J. Phys. Chem. C 2014, $118,6586$.

[74] Chen, Z.; Qin, Y.; Ren, Y.; Lu, W.; Orendorff, C. E.; Roth, P.; Amine, K. Energy Environ. Sci. 2011, 4, 4023.

[75] Wong, D. H. C.; Thelen, J. L.; Fu, Y.; Devaux, D.; Pandya, A. A.; Battaglia, V. S.; Balsara, N. P.; Desimone, J. M. PNAS 2014, 111, 3327.

[76] Arai, J. J. Electrochem. Soc. 2003, 150, A219.

[77] Naoi, K.; Iwama, E.; Ogihara, N.; Nakamura, Y.; Segawa, H.; Ino, Y. J. Electrochem. Soc. 2009, 156, A272.

[78] Naoi, K.; Iwama, E.; Honda, Y.; Shimodate, F. J. Electrochem. Soc. 2010, 157, A190. 
[79] Huang, Q.; Yan, M.-M.; Jiang, Z.-Y. Acta Chim. Sinica 2008, 66, 1. (黄倩, 严曼明, 江志裕, 化学学报, 2008, 66, 1.)

[80] Xia, L. Ph. D. Dissertation, Wuhan University, Wuhan, 2013. (夏兰, 博士论文, 武汉大学, 武汉, 2013.)

[81] Choi, J.-A.; Sun, Y.-K.; Shim, E.-G.; Scrosati, B.; Kim, D.-W. Electrochim. Acta 2011, 56, 10179.

[82] Pan, X.-R.; Lian, F.; Guan, H.-Y.; He, Y. Chemistry 2014, 77, 752. (潘笑容, 连芳, 关红艳, 何逸, 化学通报, 2014, 77, 752.)

[83] Lombardo, L.; Brutti, S.; Navarra, M. A.; Panero, S.; Reale, P. J. Power Sources 2013, 227, 8.

[84] Wang, X.; Yasukawa, E.; Kasuya, S. J. Electrochem. Soc. 2001, 148, A1058.

[85] Feng, J. K.; Ai, X. P.; Cao, Y. L.; Yang, H. X. J. Power Sources 2008, 177, 194.

[86] Jia, H.; Wang, J. L.; Lin, F. J.; Monroe, C. W.; Yang, J.; NuLi, Y. Chem. Commun. 2014, 50, 7011.

[87] Xu, K.; Ding, M. S.; Zhang, S. S.; Allen, J. L.; Jow, T. R. J. Electrochem. Soc. 2002, 149, A622

[88] Wu, B. B.; Pei, F.; Wu, Y.; Mao, R. J.; Ai, X. P.; Yang, H. X.; Cao, Y. L. J. Power Source 2013, 227, 106.

[89] Zeng, Z. Q.; Jiang, X. Y.; Wu, B. B.; Xiao, L. F.; Ai, X. P.; Yang, H. X.; Cao, Y. L. Electrochim. Acta 2014, 129, 300.

[90] Xu, K.; Ding, M. S.; Zhang, S.; Allen, J. L.; Jow, T. R. J. Electrochem. Soc. 2003, 150, A161.

[91] Xu, K.; Zhang, S. S.; Allen, J. L.; Jow, T. R. J. Electrochem. Soc. 2003, 150, A170.

[92] Tsujikawa, T.; Yabuta, K.; Matsushita, T.; Matsushima, T.; Hayashi, K.; Arakawa, M. J. Power Sources 2009, 189, 429.

[93] Zhang, S. S.; Xu, K.; Jow, T. R. J. Power Source 2003, 113, 166.

[94] Xiang, H. F.; Xu, H. Y.; Wang, Z. Z.; Chen, C. H. J. Power Source 2007, 173, 562.

[95] Xia, L.; Xia, Y.-G.; Liu, Z.-P. J. Power Sources 2015, 278, 190.

[96] Allen, C. W.; Bedell, S.; Pennington, W. T.; Cordes, A. W. Inorg. Chem. 1985, 24, 1653.

[97] Feng, J. K.; An, Y. L.; Ci, L. J.; Xiong, S. L. J. Mater. Chem. A 2015, 3, 14539 .

[98] Zhou, D.; Li, W.; Tan, C.; Zuo, X.; Huang, Y. J. Power Sources 2008, 184, 589.

[99] Arai, J.; Katayama, H.; Akahoshi, H. J. Electrochem. Soc. 2002, 149, A217.

[100] Arai, J. J. Appl. Electrochem. 2002, 32, 1071.

[101] Arai, J. J. Electrochem. Soc. 2003, 150, A219.

[102] Naoi, K.; Iwama, E.; Ogihara, N.; Nakamura, Y.; Segawa, H.; Ino, Y. J. Electrochem. Soc. 2009, 156, A272.

[103] Naoi, K.; Iwama, E.; Honda, Y.; Shimodate, F. J. Electrochem. Soc. 2010, 157, A190.

[104] Kim, G.-T.; Jeong, S. S.; Joost, M.; Rocca, E.; Winter, M.; Passerini, S.; Balducci, A. J. Power Sources 2011, 196, 2187.

[105] Kim, G.-T.; Jeong, S. S.; Xue, M.-Z.; Balducci, A.; Winter, M.; Passerini, S.; Alessandrini, F.; Appetecchi, G. B. J. Power Sources 2012,
199,239

[106] Appetecchi, G. B.; Scaccia, S.; Tizzani, C.; Alessandrini, F.; Passerini, S. J. Electrochem. Soc. 2006, 153, A1685.

[107] Kalhoff, J.; Kim, G.-T.; Passerini, S.; Appetecchi, G. B. J. Power Energy Eng. 2016, 4, 9.

[108] Yang, B.; Li, C.; Zhou, J.; Liu, J.; Zhang, Q. Electrochim. Acta 2014, $148,39$.

[109] Lombardo, L.; Brutti, S.; Navarra, M. A.; Panero, S.; Reale, P. J. Power Sources 2013, 227, 8.

[110] Wilken, S.; Xiong, S.; Scheers, J.; Jacobsson, P.; Johansson, P. J. Power Sources 2015, 275, 935.

[111] Ferrari, S.; Quartarone, E.; Mustarelli, P.; Magistris, A.; Protti, S.; Lazzaroni, S.; Fagnoni, M.; Albini, A. J. Power Sources 2009, 194, 45.

[112] Chen, Z.; Xi, H.; Lim, K. H.; Lee, J. Angew. Chem. Int. Ed. 2013, $52,13392$.

[113] Quinzeni, I.; Ferrari, S.; Quartarone, E.; Tomasi, C.; Fagnoni, M.; Mustarelli, P. J. Power Sources 2013, 237, 204.

[114] Kim, H.-T.; Kang, J.; Mun, J.; Oh, S. M.; Yim, T.; Kim, Y. G. ACS Sustainable Chem. Eng. 2016, 4, 497.

[115] Yamada, Y.; Yamada, A. J. Electrochem. Soc. 2015, 162, A2406.

[116] Yamada, Y.; Furukawa, K.; Sodeyama, K.; Kikuchi, K.; Yaegashi, M.; Tateyama, Y.; Yamada, A. J. Am. Chem. Soc. 2014, 136, 5039.

[117] Doi, T.; Masuhara, R.; Hashinokuchi, M.; Shimizu, Y.; Inaba, M. Electrochim. Acta 2016, 209, 219.

[118] Wang, J.; Yamada, Y.; Sodeyama, K.; Chiang, C. H.; Tateyama, Y.; Yamada, A. Nat. Commun. 2016, 7, 12032.

[119] Matsumoto, K.; Inoue, K.; Nakahara, K.; Yuge, R.; Noguchi, T.; Utsugi, K. J. Power Sources 2013, 231, 234.

[120] McOwen, D. W.; Seo, D. M.; Borodin, O.; Vatamanu, J.; Boyle, P. D.; Henderson, W. A. Energy Environ. Sci. 2014, 7, 416.

[121] Yamada, Y.; Chiang, C. H.; Sodeyama, K.; Wang, J.; Tateyama, Y.; Yamada, A. ChemElectroChem 2015, 2, 1687.

[122] Besenhard, J. O. Carbon 1976, 14, 111.

[123] Besenhard, J. O.; Winter, M.; Yang, J.; Biberacher, W. J. Power Sources 1995, 54, 228.

[124] Arakawa, M.; Yamaki, J. J. Electroanal. Chem. 1987, 219, 273.

[125] Abe, T.; Kawabata, N.; Mizutani, Y.; Inaba, M.; Ogumi, Z. J. Electrochem. Soc. 2003, 150, A257.

[126] Yamada, Y.; Yaegashi, M.; Abe, T.; Yamada, A. Chem. Commun. 2013, 49, 11194.

[127] Sodeyama, K.; Yamada, Y.; Aikawa, K.; Yamada, A.; Tateyama, Y. J. Phys. Chem. C 2014, 118, 14091.

[128] Suo, L.; Hu, Y.-S.; Li, H.; Armand, M.; Chen, L. Nat. Commun. 2013, 4, 1481.

[129] Qian, J.; Henderson, W. A.; Xu, W.; Bhattacharya, P.; Engelhard, M.; Borodin, O.; Zhang, J. G. Nat. Commun. 2015, 6, 6362.

[130] Ma, Q.; Fang, Z.; Liu, P.; Ma, J.; Qi, X.; Feng, W.; Nie, J.; Hu, Y.-S.; Li, H.; Huang, X.; Chen, L.; Zhou, Z. ChemElectroChem 2016, 3 , 531 . 